\title{
DEFORMATION AND LINKAGE OF GORENSTEIN ALGEBRAS
}

BY

\author{
ANDREW R. KUSTIN AND MATTHEW MILLER ${ }^{1}$
}

\begin{abstract}
General double linkage of Gorenstein algebras is defined. Rigidity, genericity, and regularity up to codimension six all pass across general double linkage. Rigid strongly unobstructed codimension four Gorenstein algebras which lie in different Herzog classes are produced.
\end{abstract}

Introduction. We begin by reviewing the classical structure theorems. Let $P=$ $k \llbracket X_{1}, \ldots, X_{m} \rrbracket, A=P / I$, and $\mathbf{F}$ be the minimal resolution of $A$ by free $P$-modules.

(1) If $A$ has codimension 1 and $I$ is unmixed, then $\mathbf{F}$ is given by $0 \rightarrow P \stackrel{f}{\rightarrow} P$ for some $f$ in $P$.

(2) (Hilbert, Burch [7], Schaps [28]) If $A$ has codimension 2 and is Cohen-Macaulay, then $\mathbf{F}$ is given by

$$
0 \rightarrow P^{n-1} \stackrel{X}{\rightarrow} P^{n} \stackrel{x}{\rightarrow} P
$$

where $X$ is an $n \times(n-1)$ matrix, $x_{i}=(-1)^{i+1} \Delta_{i}(X)$ and $\Delta_{i}(X)$ is the determinant formed by omitting the $i$ th row from $X$.

(3) (Buchsbaum and Eisenbud [4]) If $A$ has codimension 3 and its Gorenstein, then $\mathbf{F}$ is given by

$$
0 \rightarrow P \stackrel{\mathbf{x}^{t}}{\rightarrow} P^{n} \stackrel{X}{\rightarrow} P^{n} \stackrel{\mathbf{x}}{\rightarrow} P
$$

where $X$ is an $n \times n$ alternating matrix, $x_{i}=(-1)^{i+1} \operatorname{Pf}_{i}(X)$, and $\operatorname{Pf}_{i}(X)$ denotes the pfaffian of the alternating submatrix of $X$ formed by omitting row and column $i$.

In these structure theorems, as the codimension of $A$ increases so does the number of conditions on $A$. It is reasonable to suspect that a structure theorem for codimension four Gorenstein algebras $A$ will require that an additional hypothesis be imposed on $A$. We observe that in each case (a) there is a structure theorem, (b) all deformations are unobstructed (see Herzog [13]), and (c) each algebra $A$ is in the linkage class of a complete intersection (see Peskine and Szpiro [27] and Watanabe [30]). Historically these three facts were found independently. With hindsight we now see that (b) follows from (c), and (a) follows in turn from (b). In his thesis

Received by the editors June 10, 1982 and, in revised form, March 29, 1983. Some of the contents of this paper were presented to the Special Session on Commutative Algebra at the American Mathematical Society Meeting in Cincinnati on January 13, 1982.

1980 Mathematics Subject Classification. Primary 13C05, 13D25, 13H10; Secondary 14B07, 14D15, 14M05, 16A03.

${ }^{1}$ Both authors were supported in part by the National Science Foundation. 
Buchweitz [5] has shown that if $A$ is a reduced algebra which is in the linkage class of a complete intersection, then $A$ is "strongly unobstructed". Herzog [13] has shown how to obtain the structure theorem for "the class of $A$ " provided $A$ is "strongly unobstructed". From these observations, it appears that the correct continuation of the list of structure theorems should involve those codimension four Gorenstein algebras which are in the linkage class of a complete intersection. This paper is mainly concerned with such algebras. Since it is known that not all codimension four Gorenstein algebras are in the linkage class of a complete intersection (see Huneke [18, Example 2.5]), we are imposing a real restriction. Whether a more general structure theorem exists is open to question, and would certainly require different techniques.

In [23] we proposed a technique for constructing "big" Gorenstein ideals from "small" ones (size being measured by number of generators). We have since seen that the big from small construction may be viewed as linkage, or more generally, what we have come to call linkage by Gorenstein ideals. In $\$ 1$ we define linkage by Gorenstein ideals and prove the elementary facts about it. In $\$ 2$ we establish the connection between linkage by Gorenstein ideals and the big from small construction. \$\$3-7 are concerned only with ordinary linkage by complete intersections, especially in the context of general double links (Definition 2.5). In $\$ 3$ we iterate the big from small construction to produce codimension four Gorenstein $k$-algebras which we have called $A(l, \tau)$ and $B(\tau)$. §§4-7 are devoted primarly to proving results about these algebras. Generally, such results read: "if the Gorenstein algebra $A$ has property $\mathcal{P}$, then the general double link of $A$ also has $\mathcal{P} . "$

If $A$ and $B$ are complete local $k$-algebras, then Herzog [13] writes $A \sim B$ if there is a third such algebra $C$ containing regular sequences $\mathbf{x}$ and $\mathbf{y}$ such that $A \cong C /(\mathbf{x})$ and $B \cong C /(\mathbf{y})$. This relation becomes an equivalence when the algebras involved are "strongly unobstructed"; we have called the equivalence classes "Herzog classes". The calculation of four resolution invariants (Definition 7.6) allows us to conclude that the algebras $A(l, \tau)$ and $B(\tau)$ are in distinct Herzog classes.

In $\S 8$ we exhibit a codimension four Gorenstein algebra that is not in the linkage class of a complete intersection in order to show that linkage by Gorenstein ideals does not preserve the deformation-theoretic properties that ordinary linkage preserves.

Notation and conventions. Throughout this paper $k$ is a field (often algebraically closed), all rings are commutative and noetherian, and $g$ is a positive integer, usually at least 3. If $Y$ is a matrix containing entries $y_{i j}$ that are indeterminates, then $k \llbracket Y \rrbracket$ means $k \llbracket\left\{y_{i j}\right\} \rrbracket$. The symbol a or [a] represents a row vector and (a) is the ideal generated by the entries of a. In particular, $[c+v a]$ is the row vector $\left[c_{1}+\right.$ $\left.v a_{1}, \ldots, c_{n}+v a_{n}\right]$.

Let $I$ be a proper ideal of the ring $R$ with finite projective dimension. The grade of $I$ is the length of a maximal $R$-sequence contained in $I$. The codimension of $I$ (or the codimension of $R / I$ ) is $\operatorname{dim} R-\operatorname{dim} R / I$. If $R$ is Cohen-Macaulay then the grade of $I$ is equal to the height of $I$; if $R$ is also local, then both concepts coincide with the codimension of $I$. The ideal $I$ is called perfect if grade $I=p d_{R}(R / I)$. A perfect ideal 
$I$ of grade $g$ is a Gorenstein ideal if $\operatorname{Ext}_{R}{ }_{R}(R / I, R)$ is a cyclic $R / I$-module. It follows from Bass [3, Proposition 5.1] that if $I$ is a Gorenstein ideal in a Gorenstein ring $R$, then $R / I$ is also a Gorenstein ring.

A differential, graded, commutative algebra (DG) structure on a complex of free $R$-modules

$$
\mathbf{F}: \cdots \rightarrow F_{2} \stackrel{d_{2}}{\rightarrow} F_{1} \stackrel{d_{1}}{\rightarrow} F_{0}=R
$$

is a product map $\mathbf{F} \otimes_{R} \mathbf{F} \rightarrow \mathbf{F}$ with:

(1) $x_{i} x_{j} \in F_{i+j}$,

(2) $d\left(x_{i} x_{j}\right)=\left(d x_{i}\right) x_{j}+(-1)^{i} x_{i} d x_{j}$,

(3) $x_{i} x_{j}=(-1)^{i j} x_{j} x_{i}$, and

(4) $x_{i} x_{i}=0$ if $i$ is odd

for $x_{i} \in F_{i}$ and $x_{j} \in F_{j}$. Note that multiplication by $F_{0}=R$ makes $\mathbf{F}$ an $R$-algebra. If $F$ is a resolution, then by Buchsbaum and Eisenbud [4, Proposition 1.1], F has a DG structure which is homotopy associative. There are several situations in which the resolution $F$ is known to have an associative DG structure, see e.g. [4, 22]; but on the other hand for each $g \geqslant 6$ Avramov [2] has produced an example of a grade $g$ Gorenstein ideal in a Gorenstein local ring $R$ such that the minimal free resolution of $R / I$ does not admit an associative DG structure. While this issue is not critical in the present work, we would like to know if the class of Gorenstein algebras studied here also has "well-behaved" DG structures.

Question. Let $A$ be a Gorenstein quotient of $R=k \llbracket X_{1}, \ldots, X_{n} \rrbracket$. Suppose that $A$ is the linkage class of a complete intersection. Does the minimal free resolution of $A$ admit an associative DG structure?

Let $R$ be a local ring with residue field $k$, let $I$ be an ideal in $R$, and let $\mathbf{F}$ be a free resolution of $R / I$. Two of the invariants used in $\$ 7$ involve the homology algebra $\Lambda_{\bullet}=\operatorname{Tor}_{\bullet}^{R}(R / I, k)$. For a complete treatment of the algebra structure on $\Lambda_{\bullet}$ see $[8$, $\mathrm{XI}, \S \S 1-4]$. For our purposes it suffices to say that any comparison map from the complex $\mathbf{F} \otimes_{R} \mathbf{F}$ to the resolution $\mathbf{F}$ which covers the multiplication map $(R / I)$ $\otimes_{R}(R / I) \rightarrow R / I$ induces the algebra structure on $\Lambda_{\bullet}$. In particular, any (not necessarily associative) DG structure on $F$ induces the algebra structure on $\Lambda_{6}$.

We will have many occasions to use the technique that Hochster has called "general grade reduction". The main result is

Proposition A. Let a be a $1 \times n$ vector which generates a grade $g$ ideal in a noetherian ring $R$ and let $X$ be an $n \times m$ matrix of indeterminates.

(1) If $m \leqslant g$, then a $X$ is a regular sequence in $R[X]$ or $R \llbracket X \rrbracket$.

(2) If $R$ is a domain and $m \leqslant g-1$, then (a $X)$ is a prime ideal in $R[X]$ or $R \llbracket X \rrbracket$.

Proof. The results for (a $X) \subseteq R[X]$ may be found in many places, for example Hochster and Eagon [17, Propositions 21 and 22] or Hochster [15]. Statement (1) is also true for $R \llbracket X \rrbracket$ because the inclusion map $R[X] \rightarrow R \llbracket X \rrbracket$ is flat. Statement (2) is true in $R \llbracket X \rrbracket$ because the prime ideal (a $X)$ is homogeneous in $R[X]$. 
Deformation theory supplies some of our most useful tools. We recall the basic definitions and results. Artin [1] is an excellent reference. For this discussion $k$ is an algebraically closed field and $k^{\prime}=k[\varepsilon] /\left(\varepsilon^{2}\right)$ is the ring of dual numbers.

Definition. Let $A$ be a finite dimensional local $k$-algebra (note that $A / \mathrm{m}_{A}=k$ ) and suppose $B$ is a $k$-algebra. An (infinitesimal) deformation of $B$ to $A$ is a flat $A$-algebra $B^{\prime}$ such that $B^{\prime} \otimes_{A} k \cong B$. If an algebra $B^{\prime}$ is flat over $A=k \llbracket \varepsilon_{1}, \ldots, \varepsilon_{s} \rrbracket$ and $B^{\prime} \otimes_{A} k \cong B$, we say that $B^{\prime}$ is a (formal) deformation of $B$ to $A$.

Proposition B. Let $P=k \llbracket X_{1}, \ldots, X_{q} \rrbracket$ and $B=P / J$.

(1) If $\theta: k \llbracket \varepsilon_{1}, \ldots, \varepsilon_{s} \rrbracket \rightarrow B$ is a k-algebra map sending $\varepsilon_{i}$ to $b_{i}$, then $\mathbf{b}=\left[b_{1}, \ldots, b_{s}\right]$ is a regular $B$-sequence if and only if $B$ is a deformation of $B /(\mathbf{b})$ to $k \llbracket \varepsilon_{1}, \ldots, \varepsilon_{s} \rrbracket$.

(2) Let $P^{\prime}=P \otimes_{k} k^{\prime}$. Suppose $J^{\prime}$ is an ideal in $P^{\prime}$ such that $\left(P^{\prime} / J^{\prime}\right) \otimes_{k^{\prime}} k=B$. Let $\mathbf{F}^{\prime}$ be a presentation of $P^{\prime} / J^{\prime}$ by free $P^{\prime}$-modules. Then $P^{\prime} / J^{\prime}$ is a deformation of $B$ to $k^{\prime}$ if and only if $\mathbf{F}^{\prime} \otimes_{k^{\prime}} k$ is a free presentation of $B$.

(3) If $B=P / J$ has a presentation

$$
P^{m} \stackrel{d_{2}}{\rightarrow} P^{n} \stackrel{d_{1}}{\rightarrow} P
$$

and $\operatorname{Im}\left(\xi d_{2}\right) \subset J=\operatorname{Im}\left(d_{1}\right)$ for a map $\xi: P^{n} \rightarrow P$, then $P^{\prime} / J^{\prime}$ is a deformation of $B$ to $k^{\prime}$, where $J^{\prime}=\operatorname{Im}\left(d_{1}+\varepsilon \xi\right)$.

Proof. For (1) see Hartshorne [11], or argue directly using the local criterion for flatness [26, Theorem 49]. For (2) and (3) see [1].

Definition. A deformation $B^{\prime}$ of $B$ to $A$ is trivial if there is an $A$-algebra isomorphism $\theta: B \otimes_{k} A \rightarrow B^{\prime}$ such that $\theta$ induces the identity on the special fiber, i.e., $\theta \otimes 1_{k}=1_{B}$. We say $B$ is rigid if every infinitesimal deformation is trivial.

In fact, it suffices to consider the first order deformations to $k^{\prime}$. The following is a useful test criterion for rigidity.

Proposition C. Let $P=k \llbracket X_{1}, \ldots, X_{q} \rrbracket$ and $B=P / I$. Let

$$
P^{m} \stackrel{d_{2}}{\rightarrow} P^{n} \stackrel{d_{1}}{\rightarrow} P
$$

be a fixed minimal presentation of $B$, and let $q=\left(\partial f_{i} / \partial X_{j}\right)_{n \times q}$ be the Jacobian matrix for $d_{1}=\left[f_{1}, \ldots, f_{n}\right]$. Suppose $\xi: P^{n} \rightarrow P$ represents a deformation of $B$ to $k^{\prime}$ as in (3) above. The following conditions are equivalent.

(1) The deformation represented by $\xi$ is trivial.

(2) There is a $k^{\prime}$-algebra automorphism of $P^{\prime}$ which sends $J=\operatorname{Im}\left(d_{1}\right)$ to $J^{\prime}=$ $\operatorname{Im}\left(d_{1}+\varepsilon \xi\right)$, and which induces the identity on $P=P^{\prime} \otimes_{k^{\prime}} k$.

(3) There exist maps $f: P^{q} \rightarrow P$ and $g: P^{n} \rightarrow P^{n}$ such that $\xi=f g^{t}+d_{1} g$; i.e., $\xi^{t}$ is in the column space of $\mathcal{G}$, modulo $J$.

Furthermore, the algebra $B$ is rigid if every $\xi: P^{n} \rightarrow P$ as in Proposition $\mathrm{B}(3)$ satisfies these conditions.

Our final result is part of the folklore of deformation theory and can be established by using Taylor's theorem. 
Proposition D. If $A$ is a rigid $k$-algebra and $B$ is a formal deformation of $A$, then $B \cong A \llbracket Y_{1}, \ldots, Y_{s} \rrbracket$.

1. Linkage by Gorenstein ideals. The starting point of the theory of algebraic linkage is the following theorem found in Peskine and Szpiro [27, Propositions 1.3 and 2.6] or Buchsbaum and Eisenbud [4, Propositions 5.1 and 5.2].

THEOREM 1.1. Let I be a perfect ideal of grade $g$ in a Gorenstein local ring $R$ and let $K$ be an ideal properly contained in I which is generated by a length $g R$-sequence. If $J=K: I$, then:

(1) $J$ is a perfect ideal of grade $g$, and

(2) $K: J=I$.

Moreover, if $\mathbf{F}$ and $\mathbf{G}$ are minimal free resolutions of $R / I$ and $R / K$, respectively, and $\psi: \mathbf{G} \rightarrow \mathbf{F}$ is any map induced by the inclusion $K \subset I$, then the mapping cone of the dual of $\psi$ is a free resolution of $R / J$. In particular:

(3) if I and a Gorenstein ideal, then $J=(K, w)$ for some $w$, and

(4) if I is minimally generated by $(K, w)$, for some $w$, then $J$ is a Gorenstein ideal.

We note that in pathological instances, the ideal $J$ in (3) might not actually be an almost complete intersection. For example, if $K=\left(X^{2}\right)$ and $I=(X)$, then $J=$ $(K, X)=(X)$.

THEOREM 1.2. The conclusions of Theorem 1.1 remain valid if $K$ is only assumed to be a grade $g$ Gorenstein ideal properly contained in $I$.

Proof. Results (1) and (2) follow from [27, Proposition 1.3]. The proof that the mapping cone of the dual of $\psi$ resolves $R / J$ is essentially contained in [20, Proposition 1.1]. Finally, (3) and (4) follow from the mapping cone construction of the resolution of $R / J$ exactly as in the proof of [4, Proposition 5.2].

REMARK. Golod [10] has proved that (1) and (2) hold if $I$ is perfect of grade $g$ in a commutative noetherian ring $R$, and $K$ is a perfect ideal of grade $g$ properly contained in $I$. If $K$ is a Gorenstein ideal and $R$ is local, then (3) and (4) also follow. Since we are interested here in the factor rings (rather than just homological properties of the ideals) we shall retain the Gorenstein hypothesis on $R$.

Theorem 1.2 motivates the following definitions. Throughout this paper we retain the traditional meaning for the term "linked", namely "linked by a complete intersection".

DeFinITION 1.3. Let $I, J$ and $K$ be perfect ideals of the same grade in a Gorenstein local ring $R$ with $K$ properly contained in each of $I$ and $J, K: I=J$, and $K: J=I$.

(1) If $K$ is generated by a regular $R$-sequence then $I$ and $J$ are (algebraically) linked by $K$.

(2) If $K$ is a Gorenstein ideal, then $I$ and $J$ are linked by the Gorenstein ideal $K$.

DEFINITION 1.4. Two ideals $I_{0}$ and $I$ are in the same linkage class if there are ideals $I_{1}, \ldots, I_{n}$ with $I_{n}=I$ and $I_{i}$ linked to $I_{i+1}$ for $0 \leqslant i \leqslant n-1$. If $n=2$ we say $I_{0}$ and $I$ are doubly linked. 
Definition 15. An algebra $A$ is in the linkage class of a complete intersection if $A$ can be written as $R / I$, where $R$ is a regular local ring and $I$ is in the same linkage class as an ideal generated by a regular $R$-sequence. (Note that if $A=R / I \simeq R^{\prime} / I^{\prime}$, where both $R$ and $R^{\prime}$ are formal power series rings over a field $k$, then $I$ is in the linkage class of an ideal generated by a regular sequence if and only if the same holds for $I^{\prime}$.)

2. The "big from small" construction as linkage by Gorenstein ideals. Throughout this section we assume $R$ is a Gorenstein local ring and a is a $1 \times n$ matrix whose entries minimally generate a grade $g$ Gorenstein ideal of $R$. (Nothing of interest occurs unless $g \geqslant 3$.) The $n \times m$ matrix $X$ is composed of elements from $R$ and the ideal generated by the entries of the $1 \times m$ matrix $\mathbf{b}=\mathbf{a} X$ minimally generate a grade $g-1$ Gorenstein ideal of $R$. The minimal resolutions of $R /(\mathrm{a})$ and $R /(\mathrm{b})$ by free $R$-modules are denoted by $\mathbf{F}$ and $\mathbf{G}$, respectively. Buchsbaum and Eisenbud [4] observed that $\mathbf{F}$ may be given the structure of a (not necessarily associative) DG algebra and that the multiplication map $F_{i} \otimes F_{g-i} \rightarrow F_{g}=R$ is a perfect pairing. Hence, if $e_{1}, \ldots, e_{n}$ is a basis for $F_{1}$ and $g \geqslant 3$, then we can choose a basis $f_{1}, \ldots, f_{n}$ for $F_{g-1}$ so that

$$
e_{i} f_{j}=\delta_{i j}
$$

As a consequence, $\mathbf{F}$ has the form

$$
\mathbf{F}: 0 \rightarrow R \stackrel{\mathbf{a}^{t}}{\rightarrow} R^{n} \rightarrow \cdots \rightarrow R^{n} \stackrel{\mathrm{a}}{\rightarrow} R .
$$

Let $\mathbf{b}^{\prime t}$ denote the last map of $\mathbf{G}$. If $g-1 \geqslant 3$ we may assume that the vectors $\mathbf{b}^{\prime}$ and b are equal. In any case they generate the same ideal of $R$. We take $\mathbf{c}$ to denote any $1 \times n$ matrix which fits into the following comparison of minimal resolutions:

$$
\begin{aligned}
& \text { G } \quad 0 \quad \rightarrow \quad R \quad \stackrel{\mathbf{b}^{\prime \prime}}{\rightarrow} \quad \cdots \quad \rightarrow \quad R^{m} \stackrel{\text { b }}{\rightarrow} R \\
& \downarrow \quad \downarrow \mathbf{c}^{t} \quad \downarrow X \quad \| \\
& \text { F } \quad 0 \quad \rightarrow \quad R \stackrel{\stackrel{\mathrm{a}^{t}}{\rightarrow}}{\rightarrow} R^{n} \quad \rightarrow \quad \cdots \quad \rightarrow \quad R^{n} \stackrel{\mathrm{a}}{\rightarrow} \quad R
\end{aligned}
$$

Let $v$ be an indeterminate and $K$ be the ideal generated by the entries of the vector [b,c $+v \mathbf{a}$ ]. The main result of [23] is that $K$ is a grade $g$ Gorenstein ideal in $R[v]$ (and hence also in $R \llbracket v \rrbracket$ ). The main result of this section is that $K$ is obtained from (a) as a double link by Gorenstein ideals.

THEOREM 2.1. Let $R, \mathbf{a}, \mathbf{b}, \mathbf{c}$ be as described above (see diagram (2.2)). Then the ideal $K=(\mathbf{b}, \mathbf{c}+v \mathbf{a})$ is doubly linked to (a) in $R \llbracket v \rrbracket$ by Gorenstein ideals.

Proof. The ideals (b) and (0) are Gorenstein, hence unmixed, and they have grade less than the grade of (a); thus there is an element $y$ in (a) which is regular on both $R$ and $R /(\mathbf{b})$. It follows that $(\mathbf{b}, y)$ is a grade $g$ Gorenstein ideal. A minimal resolution $\mathbf{K}$ of $R /(\mathbf{b}, y)$ may be obtained by tensoring the minimal resolution of $R /(\mathbf{b})$ in $(2.2)$ with the resolution $0 \rightarrow R \stackrel{y}{\rightarrow} R$. We can extend the comparison map of (2.2) on the 
complementary summand of $\mathbf{G}$ in $\mathbf{K}$ to obtain:

$$
\begin{aligned}
& \text { G: } \quad 0 \quad \rightarrow \quad R \quad \stackrel{\mathbf{b}^{\prime \prime}}{\rightarrow} \quad \cdots \quad \rightarrow \quad R^{m} \stackrel{\mathbf{b}}{\rightarrow} R \\
& \downarrow \quad \downarrow\left[\begin{array}{c}
0 \\
\vdots \\
0 \\
1
\end{array}\right] \quad \downarrow\left[\begin{array}{l}
l \\
0
\end{array}\right] \quad \| \\
& \mathbf{K}: \quad 0 \quad \rightarrow \quad R \quad \stackrel{\left[\mathbf{b}^{\prime}, y\right]^{t}}{\rightarrow} R^{m+1} \quad \rightarrow \quad \cdots \quad \rightarrow \quad R^{m+1} \stackrel{[\mathbf{b}, y]}{\rightarrow} R \\
& \alpha_{g} \downarrow \quad \alpha_{g-1} \downarrow \quad \alpha_{1} \downarrow \quad \| \\
& \text { F: } \quad 0 \quad \rightarrow \quad R \quad \stackrel{a^{t}}{\rightarrow} \quad R^{n} \quad \rightarrow \quad \cdots \quad \rightarrow \quad R^{n} \stackrel{\text { a }}{\rightarrow} \quad R
\end{aligned}
$$

Observe that the first $m$ columns of $\alpha_{1}$ are the matrix $X$ and $\mathbf{c}^{t}$ is the last column of $\alpha_{g-1}$.

If $J=(\mathbf{b}, y)$ : (a), then by the construction of Theorem 1.2, $J=(\mathbf{b}, y, w)$ where $w=\alpha_{g}$. The element $w+v y$ of $R \llbracket v \rrbracket$ is regular on $R \llbracket v \rrbracket /(\mathbf{b})$ because $y$ is regular on $R /(\mathbf{b})$; hence $(\mathbf{b}, w+v y)$ is a grade $g$ Gorenstein ideal of $R \llbracket v \rrbracket$. If $K=(\mathbf{b}, w+v y)$ : $J$, then $K$ is obtained from (a) as a double link by Gorenstein ideals:

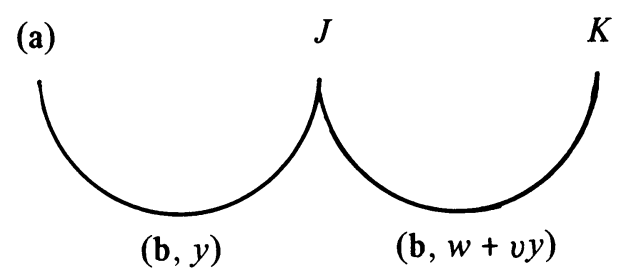

It remains to show that $K=(\mathbf{b}, \mathbf{c}+v \mathbf{a})$.

The lower right-hand commutative square of (2.3) shows that

$$
w a_{i} \equiv y c_{i} \bmod (\mathbf{b})
$$

for all $i$ since $\alpha_{g}=w$ and the last column of $\alpha_{g-1}$ is equal to $\mathbf{c}^{t}$. Thus $y\left(c_{i}+v a_{i}\right)-$ $a_{i}(w+v y)$ is in (b). Consequently, $\left(c_{i}+v a_{i}\right) y$ is in $(\mathbf{b}, w+v y)$, and $(\mathbf{b}, \mathbf{c}+v \mathbf{a})$ is contained in $(\mathbf{b}, w+v y): y=K$. On the other hand, suppose $z \in K$; so $z y-$ $s(w+v y)$ is in (b) for some $s$. Then $s w$ is in $(\mathbf{b}, y)$, and since $(\mathbf{a})=(\mathbf{b}, y): w$ by Theorem 1.2, $s=\sum s_{i} a_{i}$ and $z y-\sum s_{i}\left(a_{i} w+a_{i} v y\right)$ is in (b). It follows that $z$ is in $(\mathbf{b}, \mathbf{c}+v \mathbf{a})$ since $(\mathbf{b}, y)$ is a regular sequence.

While this theorem is useful in the form we have stated, especially for constructing explicit examples, it is too restrictive when we come to consider linkage classes of $k$-algebras. We would like, at the very least, to base the construction of $K$ on the ideal $I=(\mathbf{a})$, rather than on a particular set of generators a and resolution $\mathbf{F}$. In fact, we ultimately would like to construct a well-defined $k$-algebra $B=R \llbracket v \rrbracket / K$ starting from a $k$-algebra $A=R / I$, but without having $B$ depend on the chosen presentation of $A$. We cannot, however, change all the data with impunity. 
REMARK 2.2. (a) If $\mathbf{a}$ and $\mathbf{F}$ are fixed, then the construction of $K=(\mathbf{b}, \mathbf{c}+v \mathbf{a})$ depends on the choice of $\mathbf{c}$ in (2.2). If $\mathbf{c}^{\prime}$ is any other choice for $\mathbf{c}$, then by the homotopy equivalence of comparison maps

$$
\mathbf{c}^{\prime}=\mathbf{c}+r \mathbf{a}+\mathbf{b} \phi
$$

for a suitable matrix $\phi$ and constant $r$. The $R$-algebra automorphism of $R[v]$ which sends $v$ to $(v+r)$ carries the ideal $(\mathbf{b}, \mathbf{c}+v \mathbf{a})$ to $\left(\mathbf{b}, \mathbf{c}^{\prime}+v \mathbf{a}\right)$. The analogous statement is false in $R \llbracket v \rrbracket$, as the following example shows.

Let $R=k \llbracket A_{1}, A_{2}, A_{3} \rrbracket, \mathbf{a}=\left[A_{1}, A_{2}, A_{3}\right], \mathbf{b}=\left[A_{1}^{2}, A_{2}^{2}\right], \mathbf{b}^{\prime}=\left[-A_{2}^{2}, A_{1}^{2}\right]$,

$$
X=\left[\begin{array}{cc}
A_{1} & 0 \\
0 & A_{2} \\
0 & 0
\end{array}\right], \quad A=\left[\begin{array}{ccc}
0 & A_{3} & -A_{2} \\
-A_{3} & 0 & A_{1} \\
A_{2} & -A_{1} & 0
\end{array}\right] .
$$

Either $\mathbf{c}^{\prime}=\left[0,0, A_{1} A_{2}\right]$ or $\mathbf{c}^{\prime \prime}=\left[A_{1}, A_{2}, A_{3}+A_{1} A_{2}\right]$ may be used for $\mathbf{c}$ in:

$$
\begin{aligned}
& R \quad \stackrel{\stackrel{\mathbf{b}^{\prime}}{\rightarrow}}{\rightarrow} R^{2} \stackrel{\text { b }}{\rightarrow} R \\
& \downarrow \mathrm{c}^{t} \quad \downarrow X \quad \| \\
& R \stackrel{\stackrel{\mathbf{a}^{t}}{\rightarrow}}{\rightarrow} R^{3} \stackrel{A}{\rightarrow} \quad R^{3} \stackrel{\mathrm{a}}{\rightarrow} \quad R
\end{aligned}
$$

The ideal $\left(\mathbf{b}, \mathbf{c}^{\prime}+v \mathbf{a}\right)$ in $R \llbracket v \rrbracket$ is minimally generated by $\left(A_{1}^{2}, A_{2}^{2}, v A_{1}, v A_{2}, A_{1} A_{2}+\right.$ $v A_{3}$ ), the five maximal order pfaffians of

$$
\left[\begin{array}{ccccc}
0 & -v & 0 & A_{2} & 0 \\
v & 0 & -A_{1} & 0 & 0 \\
0 & A_{1} & 0 & A_{3} & -A_{2} \\
-A_{2} & 0 & -A_{3} & 0 & A_{1} \\
0 & 0 & A_{2} & -A_{1} & 0
\end{array}\right] .
$$

The ideal $\left(\mathbf{b}, \mathbf{c}^{\prime \prime}+v \mathbf{a}\right)$ in $R \llbracket v \rrbracket$ is

$$
\left(A_{1}^{2}, A_{2}^{2},(v+1) A_{1},(v+1) A_{2}, A_{1} A_{2}+(v+1) A_{3}\right) .
$$

Since $v+1$ is a unit this ideal is actually generated by the regular sequence $A_{1}, A_{2}$, $A_{3}$.

(b) Suppose $\mathbf{a}^{\prime}$ is another minimal set of generators for $I=(\mathbf{a})$ and $\mathbf{F}^{\prime}$ is a minimal free resolution of $R / I$ satisfying (2.1) with first map $\mathbf{a}^{\prime}$ and last map $\mathbf{a}^{\prime t}$. If $\Psi: \mathbf{F} \rightarrow \mathbf{F}^{\prime}$ is an isomorphism that lifts the identity in degree 0 , and we set $\mathbf{c}^{\prime t}=\psi_{g-1} \mathrm{c}^{t}$ and $u=\psi_{g}$, then the $R$-automorphism of $R \llbracket v \rrbracket$ given by sending $v$ to $u^{-1} v$ induces an $R$-isomorphism from $R \llbracket v \rrbracket /(\mathbf{b}, \mathbf{c}+v \mathbf{a})$ to $R \llbracket v \rrbracket /\left(\mathbf{b}, \mathbf{c}^{\prime}+v \mathbf{a}^{\prime}\right)$.

Note that in this context if $\mathbf{F}^{\prime}$ differs from $\mathbf{F}$ only at the internal maps (i.e. $\mathbf{a}^{\prime}=\mathbf{a}$ ) and $\mathbf{c}^{\prime t}$ satisfies (2.2) with $\mathbf{F}^{\prime}$ in place of $\mathbf{F}$, then

$$
\mathbf{c}^{\prime}=\mathbf{c} \psi+r \mathbf{a}+\mathbf{b} \phi
$$

where $\psi=\psi_{g-1}^{t}$ is invertible, $u \mathbf{a}=\mathbf{a} \psi, \phi$ is a matrix, $u$ is a unit, and $r$ is a constant. Henceforth we shall allow this harmless indeterminacy in $\mathbf{F}$ whenever we refer to diagram (2.2). 
If we link by complete intersections, then we can specify how to choose $\mathbf{c}$ so that the construction of $R \llbracket v \rrbracket / K$ is canonical. To this end, observe that if $b_{1}, \ldots, b_{g-1}$ are elements of $R$, then $\mathbf{K}(\mathbf{b})$, the Koszul complex on $\mathbf{b}$, is an exterior algebra. By [4], $\mathbf{F}$ has a (not necessarily associative) DG structure. Select $\varepsilon_{1}, \ldots, \varepsilon_{g-1}$ and $e_{1}, \ldots, e_{n}$ bases for $K_{1}$ and $F_{1}$, respectively. It is easy to see that the map $\alpha: \mathbf{K}(\mathbf{b}) \rightarrow \mathbf{F}$, defined for all $1 \leqslant j_{1}<\cdots<j_{r} \leqslant g-1$ by

$$
\alpha\left(\varepsilon_{j_{1}} \varepsilon_{j_{2}} \cdots \varepsilon_{j_{r}}\right)=\sum_{1 \leqslant i_{1}<i_{2}<\cdots<i_{r} \leqslant n} e_{i_{1}}\left(e_{i_{2}}\left(\cdots\left(e_{i_{r}}\right) \cdots\right)\right) X\left(i_{1}, \ldots, i_{r} ; j_{1}, \ldots, j_{r}\right),
$$

is a map of complexes, where $X\left(i_{1}, \ldots, i_{r} ; j_{1}, \ldots, j_{r}\right)$ denotes the determinant of the submatrix of $X$ formed by the rows $i_{1}, \ldots, i_{r}$ and column $j_{1}, \ldots, j_{r}$. Under this definition and the pairing $(2.1), \mathbf{c}^{t}=\left[c_{1}, \ldots, c_{n}\right]$ is given by

$$
c_{l}=e_{l} \alpha\left(\varepsilon_{1} \cdots \varepsilon_{g-1}\right) \text {. }
$$

If we impose a rather minimal hypothesis on the linking matrix $X$, then any vector $\mathbf{c}^{\prime}$ which completes (2.2) and one other condition gives rise to the same algebra $R \llbracket v \rrbracket / K$.

Proposition 2.3. In addition to the general hypotheses of this section, assume that $\mathbf{b}$ is a regular sequence and that the entries $x_{1 i}, x_{2 i}, \ldots, x_{n i}$ of one of the columns of $X$ generate an ideal I with the property that (a) is not contained in (b) $+I$. Let $\mathbf{c}$ be defined by (2.7) and $\mathbf{c}^{\prime}$ be any other vector which satisfies (2.2) and is contained in $I$. Then $R \llbracket v \rrbracket /(\mathbf{b}, \mathbf{c}+v \mathbf{a})$ is isomorphic to $R \llbracket v \rrbracket /\left(\mathbf{b}, \mathbf{c}^{\prime}+v \mathbf{a}\right)$ as an $R$-algebra.

REMARK. Our typical application is in the situation that (a) $\subset R$, and the nonzero entries of one column of $X$ indeterminates $X_{1}, \ldots, X_{r}$ over $R$. We replace $R$ by $R \llbracket X_{1}, \ldots, X_{r} \rrbracket$ and take $I=\left(X_{1}, \ldots, X_{r}\right)$. If (a) $\subset$ (b) $+I$, then by setting all $X_{i}$ equal to 0 we would have (a) $\subset$ (b), a contradiction to $\operatorname{grade}(\mathbf{a})=g$.

Proof. Here $\mathbf{G}=\mathbf{K}$ is the Koszul resolution of $R /(\mathbf{b})$. By (2.6), $\mathbf{c}^{\prime}=\mathbf{c} \psi=r \mathbf{a}+\mathbf{b} \phi$, where $\psi$ is an invertible matrix, $\mathbf{a} \psi=u \mathbf{a}$ for some unit $u$, and $\phi$ is a matrix. Thus $\psi=u I+d_{2} \psi^{\prime}$ for some matrix $\psi^{\prime}$. Duality of resolutions of Gorenstein algebras implies $\mathbf{c} d_{2}=\mathbf{b} \boldsymbol{\theta}$ for some $\boldsymbol{\theta}$; thus $\mathbf{c}^{\prime}=u \mathbf{c}+r \mathbf{a}+\mathbf{b} \boldsymbol{\theta}^{\prime}$ for some $\boldsymbol{\theta}^{\prime}$. If $r$ were a unit, then (a) would be contained in (b) $+I$ since $\left(\mathbf{c}^{\prime}\right) \subset I$ by hypothesis and (c) $\subset I$ by expansion of each $X((j): 1, \ldots, g-1)$ down the $i$ th column. We conclude $r$ is in $m_{R}$ and the $R$-algebra isomorphism of $R \llbracket v \rrbracket$ defined by sending $v$ to $(v+r) u^{-1}$ carries $(\mathbf{b}, \mathbf{c}+v \mathbf{a})$ to $\left(\mathbf{b}, \mathbf{c}^{\prime}+v \mathbf{a}\right)$.

REMARK 2.4. We now assume that $X$ is an $n \times(g-1)$ matrix of indeterminates and that $R=S \llbracket X \rrbracket$, where $S$ is a Gorenstein local ring. Suppose that a and $\mathbf{a}^{\prime}$ are minimal generating sets for a codimension $g$ Gorenstein ideal $I$ in $S$ and that the respective $R$-free minimal resolutions $\mathbf{F}$ and $\mathbf{F}^{\prime}$ of $R / I R$ have the form exhibited in (2.2). Suppose that $\mathbf{c}$ and $\mathbf{c}^{\prime}$ are vectors which complete (2.2) and which have all their entries in the ideal $\left(x_{1 i}, \ldots, x_{n i}\right)$ for some fixed, but arbitrary, $i$. By Proposition A both $\mathbf{b}=\mathbf{a} X$ and $\mathbf{b}^{\prime}=\mathbf{a}^{\prime} X$ are regular sequences in $R$. Therefore we conclude that $R \llbracket v \rrbracket /(\mathbf{b}, \mathbf{c}+v \mathbf{a})$ and $R \llbracket v \rrbracket /\left(\mathbf{b}^{\prime}, \mathbf{c}^{\prime}+v \mathbf{a}^{\prime}\right)$ are isomorphic $S$-algebras. The argument is straightforward and uses Proposition 2.3 twice. This remark motivates the following definition. 
Definition 2.5. Let $A=R / I$, where $I$ is a Gorenstein ideal of grade $g$, and $R$ is a Gorenstein local ring. Assume that $g \geqslant 3$. Let a be a $1 \times n$ matrix which minimally generates $I$; let $X$ be an $n \times(g-1)$ matrix of indeterminates; and let c be a $1 \times n$ vector that completes (2.2) with (c) contained in one of the ideals $\left(x_{1 i}, \ldots, x_{n i}\right)$. Let $v$ be an indeterminate. We call $B=R \llbracket X, v \rrbracket /(\mathbf{a} X, \mathbf{c}+v \mathbf{a})$ the general double link of A.

By the preceding results, $B$ is a codimension $g$ Gorenstein quotient of $R \llbracket X, v \rrbracket$ that depends up to $R$-isomorphism only on $A$. If $A$ is itself only defined up to $k$-algebra isomorphism, the same is true of $B$. If $A$ is in the linkage class of a complete intersection so is $B$. One advantage of algebras obtained as general double links is that they can be given a natural grading.

Proposition 2.6. Adopt the same notation as in Definition 2.5. Give $R \llbracket X, v \rrbracket a$ grading by $\operatorname{deg} R=0, \operatorname{deg} x_{i j}=1$, and $\operatorname{deg} v=g-1$. Then the general double link of the R-algebra $A$ is a quotient of $R \llbracket X, v \rrbracket$ by a homogeneous ideal.

Proof. Let $\mathbf{F}$ be the minimal free resolution of $A$ over $R, \mathbf{K}$ be the Koszul resolution (with exterior algebra structure) of the complete intersection $\mathbf{R} \llbracket X, v \rrbracket /(\mathbf{a} X)$, and $\alpha: \mathbf{K} \rightarrow \mathbf{F} \otimes_{R} R \llbracket X, v \rrbracket$ be the complex map as in the discussion prior to Proposition 2.3. Each product $e_{l}\left(e_{i_{1}}\left(e_{i_{2}} \cdots\left(e_{i_{g-1}}\right)\right)\right)$ of (2.7) is in $R$ and has degree 0 . Each $X\left(i_{1}, \ldots, i_{g-1} ; 1, \ldots, g-1\right)$ is a form of degree $g-1$ in the $x_{i j}$. Since each entry of the vector $\mathbf{a}$ is in $R$, we see that each entry of the vector $\mathbf{c}+v \mathbf{a}$ is a form of degree $g-1$ and each entry of the operator $\mathbf{a} X$ is a form of degree 1 . Thus the ideal $(\mathbf{a} X, \mathbf{c}+v \mathbf{a})$ is homogeneous.

Proposition 2.7. Let $\tau \geqslant 3$ be an odd integer, $Y$ be a $\tau \times \tau$ alternating matrix with indeterminate entries, $I$ be the ideal in $k \llbracket Y \rrbracket$ generated by the maximal order pfaffians of $Y$, and $A=k \llbracket Y \rrbracket / I$. Then $A$ is obtained from a complete intersection by a series of general double links.

Proof. The proof is by induction on $\tau$. If $\tau=3$, then $A=k$ is a complete intersection. Suppose that the statement is true for $\tau$. Let $Y_{i}, Y_{i j k}$ and $Y_{i j k l m}$ be the pfaffians of $Y$ (of order $\tau-1, \tau-3, \tau-5$, respectively) as defined in [24]. Let $\mathbf{y}=\left[Y_{1}, \ldots, Y_{\tau}\right], X$ be a $\tau \times 2$ matrix of indeterminates, $\mathbf{b}=\left[b_{1}, b_{2}\right]=\mathbf{y} X$, and $\mathbf{c}$ be the $1 \times \tau$ matrix with $c_{a}=-\sum_{i<j} d_{i j} Y_{i j a}$, where $d_{i j}$ is the minor of $X$ using rows $i$ and $j$. It is straightforward to check that

$$
\begin{aligned}
& 0 \rightarrow R \quad \stackrel{\left[\begin{array}{c}
-h_{2} \\
b_{1}
\end{array}\right]}{\rightarrow} \quad R^{2} \stackrel{\left[b_{1}, h_{2}\right]}{\rightarrow} R
\end{aligned}
$$

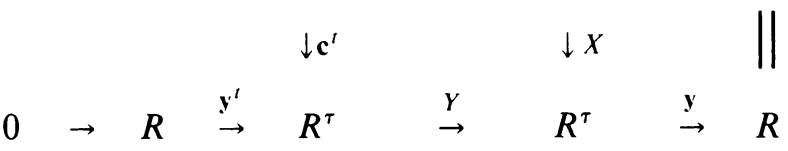

is a commutative diagram and (c) is contained in $\left(x_{11}, x_{21}, \ldots, x_{\tau 1}\right)$. We conclude that $B=k \llbracket Y, X, v \rrbracket /(\mathbf{b}, \mathbf{c}+v \mathbf{a})$ is indeed the general double link of the $k \llbracket Y \rrbracket$-algebra $A$. 
Let $M$ be the $(\tau+2) \times(\tau+2)$ alternating matrix

$$
M=\left[\begin{array}{cc|ccc}
0 & -v & -x_{12} & \cdots & -x_{\tau 2} \\
v & 0 & x_{11} & \cdots & x_{\tau 1} \\
\hline x_{12} & -x_{11} & & & \\
\vdots & \vdots & & Y & \\
x_{\tau 2} & -x_{\tau 1} & & &
\end{array}\right] .
$$

We claim that $B=k \llbracket M \rrbracket / K$, where $K$ is generated by the maximal order (i.e., $\tau+1)$ pfaffians $M_{i}$ of $M$. This is a straightforward computation using the expansion formulas of [24, Lemma 1.2].

In $\S 6$ we shall study how estimation of local number of generators can be passed over a double link. The most interesting situation occurs when $(a)_{\mathfrak{p}}$ becomes a complete intersection. We fix all notation as in Theorem 2.1 and let $S=R \llbracket v \rrbracket$. Suppose $P$ is a prime of $S$ that contains both $K$ and $J$. Let $\mathfrak{p}=P \cap R$ and $\mathbf{a}^{\prime}$ be a $1 \times g$ vector whose elements minimally generate $J_{p}$. Let $\alpha \in R \backslash \mathfrak{p}$ and $U$ be a $g \times n$ matrix with entries in $R$ such that $\alpha \mathbf{a}=\mathbf{a}^{\prime} U$. Set $X^{\prime}=U X$; then $\alpha \mathbf{b}=\alpha \mathbf{a} X=\mathbf{a}^{\prime} X^{\prime}$ generates a complete intersection inside $J_{\mathfrak{p}}$.

LEMMA 2.8 (NOTATION AS DESCRIBED ABOVE). Either $K_{P}$ is a complete intersection, or there is a $1 \times g$ vector $\mathbf{e}$ with entries in $R_{\mathfrak{p}}$ such that $K_{P}=\left(\mathbf{b}, \mathbf{e}+v \mathbf{a}^{\prime}\right)$ in $S_{P}$ and $\mathbf{e} X^{\prime}=\beta \mathbf{b}$ for some $\beta \in R_{\mathrm{p}}$.

Proof. As in the proof of Theorem 2.1, choose $y$ in $J$ that is regular on $R /(\mathbf{b})$, and let $I=(\mathbf{b}, y): J$. There is an element $w$ in $R$ such that $I=(\mathbf{b}, y, w)$ and $K=$ $(\mathbf{b}, w+v y): I$. If $I_{\mathfrak{p}}$ is a complete intersection, then $K_{P}$ has at most $g+1$ generators; consequently, it must be a complete intersection by Kunz [19]. We assume that $I_{\mathrm{p}}$ is not a complete intersection. Using the exterior algebra structures, we define a map $\theta$ of free $R_{\mathrm{p}}$-resolutions with the first $g-1$ columns of $\theta_{1}$ given by $X^{\prime}$ :

$$
\begin{aligned}
& 0 \rightarrow R_{\mathfrak{p}} \stackrel{[\alpha \mathbf{b}, y]^{t}}{\rightarrow} \quad R_{\mathfrak{p}}^{g} \quad \rightarrow \quad \cdots \quad \rightarrow \quad R_{\mathfrak{y}}^{g} \stackrel{\left[\alpha \mathbf{b}_{,}, y\right]}{\rightarrow} R_{\mathfrak{p}} \\
& \downarrow w^{\prime} \quad \downarrow \theta_{g-1} \quad \downarrow \theta_{1} \quad \| \\
& 0 \rightarrow R_{\mathfrak{p}} \stackrel{\stackrel{\left(\mathbf{a}^{\prime}\right)^{t}}{\rightarrow}}{\rightarrow} \quad R_{\mathfrak{p}}^{g} \stackrel{d_{g-1}}{\rightarrow} \quad \cdots \rightarrow \rightarrow R_{\mathfrak{p}}^{g} \stackrel{\stackrel{\mathrm{a}^{\prime}}{\rightarrow}}{\rightarrow} R_{\mathfrak{p}}
\end{aligned}
$$

If the last column of $\theta_{g-1}$ is denoted $\left(\mathbf{c}^{\prime}\right)^{t}$, then $\mathbf{c}^{\prime} X^{\prime}=0$. Now $I_{\mathfrak{p}}=\left(\mathbf{b}, y, w^{\prime}\right)$ in $R_{\mathfrak{v}}$ by Theorem 1.2, and since $w$ is not in $(\mathbf{b}, y)_{p}$, we have $w=u\left(w^{\prime}+r y+\gamma\right)$ for some $u, r, \gamma$ in $R_{\mathfrak{p}}$ with $u$ a unit and $\gamma$ in $(\mathbf{b})_{\mathfrak{p}}$. If we replace $w^{\prime}$ by $w^{\prime \prime}=w^{\prime}+r y$, and $\mathbf{c}^{\prime}$ by $\mathbf{c}^{\prime \prime}=\mathbf{c}^{\prime}+r \mathbf{a}^{\prime}$, then the diagram still commutes. The lower row can be mapped isomorphically to another resolution of $R_{\mathfrak{p}} / J_{\mathfrak{p}}$

$$
0 \rightarrow R_{\mathfrak{p}} \stackrel{\left(\mathbf{a}^{\prime}\right)^{t}}{\rightarrow} R_{\mathfrak{p}}^{g} \stackrel{u^{-1} d_{g-1}}{\rightarrow} \cdots \rightarrow R_{\mathfrak{p}}^{g} \stackrel{\mathbf{a}^{\prime}}{\rightarrow} R_{\mathfrak{p}},
$$

in effect replacing $w^{\prime \prime}$ by $u w^{\prime \prime}=w-u \gamma$ and $\mathbf{c}^{\prime \prime}$ by $\mathbf{e}=u \mathbf{c}^{\prime \prime}$. Observe that $\mathbf{e} X^{\prime}=\beta \mathbf{b}$, where $\beta=u r \alpha$ is in $R_{p}$. It is clear that $K_{P}=\left(\alpha \mathbf{b}, u w^{\prime \prime}+v y\right): I_{P}$; and since 
$u w^{\prime \prime} a_{i}^{\prime} \equiv y e_{i} \bmod (\alpha \mathbf{b})$ the last part of the proof of Theorem 2.1 also demonstrates that $K_{P}=\left(\mathbf{b}, \mathbf{e}+v \mathbf{a}^{\prime}\right)$.

REMARKs. (1) The same argument works even if $J_{\mathfrak{p}}$ is not a complete intersection, provided the resolution has an associative DG structure.

(2) The result can be viewed as showing that the phenomenon of Remark 2.2(a) is typical (if $\mathfrak{p}=\mathfrak{m}_{R}$ and (a) is already a complete intersection).

3. The $k$-algebras $A(l, \tau)$ and $B(\tau)$. Throughout this section $k$ is a fixed field with characteristic not equal to 2 . The characterization assumption is made only to guarantee that minimal free resolutions of codimension four Gorenstein $k$-algebras admit associative DG structures. We iterate the "big from small" construction of [23] to define codimension four Gorenstein $k$-algebras $A(l, \tau)$ and $B(\tau)$ and we produce their minimal free resolutions. In the notation " $l$ " represents the number of general double links and " $\tau$ " refers to the generic codimension three Gorenstein algebra with which we start.

Fix an odd integer $\tau \geqslant 3$ and let $Y$ be the $\tau \times \tau$ alternating matrix with indeterminate entries $y_{i j}$ for $i<j$. Let $J$ be the ideal generated by the maximal order pfaffians $\operatorname{Pf}_{i}(Y), 1 \leqslant i \leqslant \tau$, of $Y$ together with one more indeterminate $w$ in the ring $R(0, \tau)=k \llbracket Y, w \rrbracket$, and let $A(0, \tau)$ be the codimension four Gorenstein algebra $R(0, \tau) / J$ (see [4, Proposition 6.1]). Suppose that $A(l, \tau)$ and $R(l, \tau)$ have been defined, and that $A(l, \tau)=R(l, \tau) / I$ where $I$ is minimally generated by $n$ elements. Let $R(l+1, \tau)=R(l, \tau) \llbracket X, v \rrbracket$ where $v$ is an indeterminate and $X$ is an $n \times(g-1)$ matrix of indeterminates. Let $A(l+1, \tau)$ be the general double link of the $R(l, \tau)$-algebra $A(l, \tau)$ as found in Definition 2.5 .

To define $B(\tau)$, let $R=R(0, \tau)$, and let $\mathbf{F}$ be a minimal free resolution of $A(0, \tau)$ in which $\mathbf{a}=\left[Y_{1}, \ldots, Y_{\tau}, w\right]$, where $Y_{i}=(-1)^{i+1} \operatorname{Pf}_{i}(Y)$. Let $X$ be the $(\tau+1) \times 3$ matrix

$$
X=\left[\begin{array}{ccc}
1 & 0 & 0 \\
0 & x_{22} & x_{23} \\
\vdots & \vdots & \vdots \\
0 & x_{\tau 2} & x_{\tau 3} \\
x_{\tau+1} 1 & x_{\tau+12} & x_{\tau+13}
\end{array}\right]
$$

where the $x_{i j}$ are indeterminates. As usual $\mathbf{b}=\mathbf{a} X$ and we take $I=(\mathbf{b}, \mathbf{c}+v \mathbf{a})$ to be the ideal in $R(\tau)=R \llbracket X, v \rrbracket$ described prior to Proposition 2.3. Define $B(\tau)$ to be $R(\tau) / I$.

Note that $A(l, \tau)=R(l, \tau) / I$ is not well defined as an $R(l, \tau)$-algebra because there can be several different choices for $I=(\mathbf{b}, \mathbf{c}+v \mathbf{a})$ corresponding to different choices for c. Nonetheless, by Remark 2.4 the $k$-algebra $A(l, \tau)$ is well defined, independent of the particular presentation of $A(l-1, \tau)=R(l-1, \tau) /(\mathbf{a})$ or DG structure on its minimal free resolution. In contrast, because of the asymmetry in $X$ given by (3.1), the algebra $B(\tau)$ does depend on the particular vector a, though by virtue of Proposition 2.3 not on the particular DG structure on the minimal free resolution of $R(0, \tau) /(\mathbf{a})$. 
Proposition 3.1. Let $l \geqslant 0$ and $\tau \geqslant 3$ be integers with $\tau$ odd.

(1) The $k$-algebra $A(l, \tau)$ (respectively $B(\tau)$ ) is well defined, Gorenstein, and in the linkage class of a complete intersection. As a quotient of $R=R(l, \tau)$ (respectively $R(\tau)$ ), it has codimension four.

(2) The minimal resolution of $A(l, \tau)$ (respectively $B(\tau)$ ) by free $R$-modules admits an associative $D G$ structure.

(3) Let $\mathcal{P}$ be a property of local k-algebras. Assume that $k$ has $\mathscr{P}$ and that $\mathscr{P}$ is preserved by general double link. Then each algebra $A(l, \tau)$ has $\mathscr{P}$.

Proof. (1) It is clear that $A(0, \tau)$ is well defined, and the other properties have been noted above and in Proposition 2.7. The remarks following Definition 2.5 show that $A(l, \tau)$ have the required properties for $l \geqslant 1$.

Now consider the algebra $B(\tau)$. We first verify that $\mathbf{b}$ is a regular sequence. By Proposition A

$$
\mathbf{a}\left[\begin{array}{cc}
x_{12} & x_{13} \\
\vdots & \vdots \\
x_{\tau+1} 2 & x_{\tau+13}
\end{array}\right]
$$

generates a codimension two prime ideal. This ideal does not contain $a_{1}+a_{\tau+1} x_{\tau+1}$ 1 $=Y_{1}+w x_{\tau+1}$. By adjoining this element and making a change of variables to eliminate $x_{12}$ and $x_{13}$, we see that (b) is generated by a regular sequence of length 3 . By Theorem 2.1, the ideal (b, c $+v \mathbf{a})$ is Gorenstein, codimension four, and in the same linkage class as (a). By Proposition 2.3 the $k$-algebra $B(\tau)$ depends only on a and not on the choice of a DG structure on $\mathbf{F}$.

(2) Let $A=A(l, \tau)$ (respectively $B(\tau)$ ) and write $A=R / I$ with $R=R(l, \tau)$ (respectively $R(\tau)$ ). Since $1 / 2 \in R$ and grade $I=4$, the main theorem of [22] guarantees that the minimal free resolution of $A$ admits an associative DG structure.

(3) The algebra $A(0,3)$ is $k$. By Proposition 2.7 , the algebra $A(0, \tau+2)$ is the general double link of the $R(0, \tau) /(w)$-algebra $A(0, \tau)$; and by definition $A(l+1, \tau)$ is the general double link of the $R(l, \tau)$-algebra $A(l, \tau)$.

ExAmples 3.2. (1) Let a $=\left[A_{1}, A_{2}, A_{3}, A_{4}\right]$ be a vector of indeterminates, $X$ be a $4 \times 3$ matrix of indeterminates, and $v$ be another indeterminate. Then $R=R(1,3)$ $=k \llbracket \mathbf{a}, X, v \rrbracket$ is a power series ring in 17 variables. The vector $\mathbf{c}$ is computed by completing the following map of exterior algebras

$$
\begin{aligned}
& R \quad \stackrel{\mathbf{b}^{t}}{\rightarrow} R^{3} \stackrel{B}{\rightarrow} R^{3} \stackrel{\text { b }}{\rightarrow} R \\
& \downarrow X \quad \| \\
& R \stackrel{\mathbf{a}^{t}}{\rightarrow} R^{4} \stackrel{s A^{t}}{\rightarrow} R^{6} \stackrel{A}{\rightarrow} \quad R^{4} \stackrel{\text { a }}{\rightarrow} \quad R
\end{aligned}
$$

with

$$
B=\left[\begin{array}{ccc}
0 & b_{3} & -b_{2} \\
-b_{3} & 0 & b_{1} \\
b_{2} & -b_{1} & 0
\end{array}\right], \quad A=\left[\begin{array}{cccccc}
0 & A_{3} & -A_{2} & A_{4} & 0 & 0 \\
-A_{3} & 0 & A_{1} & 0 & A_{4} & 0 \\
A_{2} & -A_{1} & 0 & 0 & 0 & A_{4} \\
0 & 0 & 0 & -A_{1} & -A_{2} & -A_{3}
\end{array}\right]
$$


and $s=\left[\begin{array}{cc}0 & I_{3} \\ I_{3} & 0\end{array}\right]$. According to $(2.7), c_{i}=(-1)^{i+1} \Delta_{i}(X)$. The ideal $(\mathbf{a} X$, c) was first studied by Herzog in [12] and was named a "Herzog ideal" by Huneke in [18]. The algebra $A(1,3)=R(1,3) /(\mathbf{a} X, \mathbf{c}+v \mathbf{a})$ is the versal deformation of $k \llbracket \mathbf{a}, X \rrbracket /(\mathbf{a} X, \mathbf{c})$.

(2) The algebras $A(1, \tau)$ and their minimal free resolutions have been given in [24, Theorem 2.1 and 20, $\$ 4]$ where $\sigma=3$ in the notation of that paper.

(3) It is not difficult to check that $R(3) \cong R(0,5) \llbracket x_{41} \rrbracket$ and $B(3) \cong A(0,5) \llbracket x_{41} \rrbracket$.

(4) In [24, Example 4.2] we considered algebras, which we shall here denote $B^{\prime}(\tau)$, that turn out to be essentially the $B(\tau)$. In [24] we used (after a change of variables)

$$
X=\left[\begin{array}{ccc}
1 & 0 & 0 \\
x_{21} & x_{22} & x_{23} \\
\vdots & \vdots & \vdots \\
x_{\tau+11} & x_{\tau+12} & x_{\tau+13}
\end{array}\right],
$$

whereas here in constructing $B(\tau)$ we have used $X$ as in (3.1) with $x_{j 1}=0$ for $j=2, \ldots, \tau$. So it is not surprising that $B^{\prime}(\tau) \cong B(\tau) \llbracket x_{21}, \ldots, x_{\tau 1} \rrbracket$ as $k$-algebras. It is often easier to prove properties for $B^{\prime}(\tau)$ than for $B(\tau)$ due to the simpler block decomposition of $X$. The claimed isomorphism is a consequence of the following slightly more general result.

LEMMA 3.3. Let a be a $1 \times n$ matrix which minimally generates a grade $g$ Gorenstein ideal in a Gorenstein local $k$-algebra $R$. Let $X^{t}=\left[x_{2}, \ldots, x_{n}\right]$ and $Z=\left(z_{i j}\right)$, with $2 \leqslant i \leqslant n$ and $2 \leqslant j \leqslant g-1$, be matrices of indeterminates. Let $X_{1}^{t}=\left[x_{2}, \ldots, x_{r}\right]$ and $X_{2}^{t}=\left[x_{r+1}, \ldots, x_{n}\right]$ for $r$ fixed, $1 \leqslant r \leqslant n$. Set $S=R \llbracket X, Z \rrbracket, T=R \llbracket X_{2}, Z \rrbracket$,

$$
M=\left[\begin{array}{l|l}
1 & 0 \\
\hline X & Z
\end{array}\right], \quad M^{\prime}=\left[\begin{array}{l|l}
1 & 0 \\
\hline 0 & Z \\
\hline X_{2} & Z
\end{array}\right],
$$

$\mathbf{b}=\mathbf{a} M, \mathbf{b}^{\prime}=\mathbf{a} M^{\prime}$, and assume that $\mathbf{b}$ and $\mathbf{b}^{\prime}$ are regular sequences. Use (2.7) to define $\mathbf{c}\left(\right.$ respectively $\left.\mathbf{c}^{\prime}\right)$ as the last map from the Koszul resolution of $S /(\mathbf{b})\left(\right.$ resp. $\left.T /\left(\mathbf{b}^{\prime}\right)\right)$ to $\mathbf{F} \otimes S($ resp. $\mathbf{F} \otimes T)$. Let $A=S \llbracket v \rrbracket /(\mathbf{b}, \mathbf{c}+v \mathbf{a})$ and $B=T \llbracket v \rrbracket /\left(\mathbf{b}^{\prime}, \mathbf{c}^{\prime}+v \mathbf{a}\right)$. If there is a k-algebra automorphism $\theta$ of $R \llbracket X_{1} \rrbracket$ such that $\theta\left(a_{1}\right)=a_{1}+\sum_{i=2}^{r} a_{i} x_{i}$ and $\theta\left(a_{j}\right)=a_{j}$ for $j=2, \ldots, n$, then $A$ and $B \llbracket X_{1} \rrbracket$ are isomorphic $k$-algebras.

Proof. Let $\mathbf{a}^{\prime}=\theta(\mathbf{a})$ and observe that these vectors generate the same ideal in $S$. In fact, $\mathbf{a}=\mathbf{a}^{\prime} N$ and $M^{\prime}=N M$ where $N$ is the $n \times n$ invertible matrix

$$
N=\left[\begin{array}{c|c}
1 & 0 \\
\hline-X_{1} & \\
0 & I
\end{array}\right] \text {. }
$$

If we take $\psi_{1}=N, \psi_{g-1}=\left(N^{-1}\right)^{t}$, and the other $\psi_{i}=$ id, then as in Remark 2.2 we have $A=S \llbracket v \rrbracket /(\mathbf{b}, \mathbf{c}+v \mathbf{a})=S \llbracket v \rrbracket /\left(\mathbf{b}, \mathbf{d}+v \mathbf{a}^{\prime}\right)$ where $\mathbf{d}=\mathbf{c} N^{-1}$. Note that $\mathbf{d}$ and $\mathbf{c}$ satisfy the hypothesis of Proposition 2.3. We can extend $\theta$ to an automorphism of $S$ by taking it to be the identity on $X_{2}$ and $Z$. Then $\theta^{-1}(\mathbf{b})=\theta^{-1}\left(\mathbf{a}^{\prime} M^{\prime}\right)=\mathbf{a} M^{\prime}=\mathbf{b}^{\prime}$ and thus $A \cong S \llbracket v \rrbracket /\left(\mathbf{b}^{\prime}, \theta^{-1}(\mathbf{d})+v \mathbf{a}\right) \cong S \llbracket v \rrbracket /\left(\mathbf{b}^{\prime}, \mathbf{c}^{\prime}+v \mathbf{a}\right)$ by Proposition 2.3. The last algebra is precisely $B \otimes T \llbracket X_{1} \rrbracket=B \llbracket X_{1} \rrbracket$. 
In the application $r=\tau$, and $\theta$ is the automorphism of $k \llbracket Y, w, x_{21}, \ldots, x_{\tau 1} \rrbracket$ given by fixing $w$ and the $x_{i 1}$ and sending the alternating matrix $Y$ to $N Y N^{t}$, where

$$
N=\left[\begin{array}{c|c}
1 & 0 \cdots 0 \\
\hline-x_{21} & \\
\vdots & I_{\tau-1} \\
-x_{\tau_{1}} &
\end{array}\right] .
$$

One can check that $\theta\left(Y_{1}\right)=Y_{1}+\sum_{i=2}^{\tau} Y_{i} x_{i 1}$, and $\theta\left(Y_{j}\right)=Y_{j}$ for $2 \leqslant j \leqslant \tau$.

The rest of this section is devoted to expressing the minimal resolution of $A(l+1, \tau)$ in terms of the minimal resolution of $A(l, \tau)$. Lemma 3.4 is a fact about resolutions of any codimension four local Gorenstein algebra. The result is implicit in [21 and 22]. We present the most direct proof.

LEMMA 3.4. Let $A=R / I$ be a codimension four Gorenstein quotient of a local Gorenstein ring $R$. Let

$$
\mathbf{F}: 0 \rightarrow R \stackrel{d_{4}}{\rightarrow} F_{3} \stackrel{d_{3}}{\rightarrow} F_{2} \stackrel{d_{2}}{\rightarrow} F_{1} \stackrel{d_{1}}{\rightarrow} R
$$

be a (not necessarily associative) $D G$ algebra which is a minimal free resolution of $A$. If $e_{1}, \ldots, e_{n}$ is a basis for $F_{1}$, and $f_{1}, \ldots, f_{m}$ is a basis for $F_{2}$, then there is a basis $g_{1}, \ldots, g_{n}$ for $F_{3}$, and an invertible symmetric matrix s such that:

(1) $d_{4}=d_{1}^{t}$.

(2) $d_{3}=s d_{2}^{t}$.

(3) If $p=\sum p_{i} e_{i} \in F_{1}$ and $q=\sum q_{i} g_{i} \in F_{3}$ are viewed as column vectors, then $p q=\sum p_{i} q_{i}=p^{t} q$.

(4) If $p$ and $q$ in $F_{2}$ are viewed as column vectors with respect to the basis $f_{1}, \ldots, f_{m}$, then $p q=-p^{t} s^{-1} q$.

Proof. The existence of the basis $g_{1}, \ldots, g_{n}$ and assertions (1) and (3) follow from [4] as discussed at the beginning of $\S 2$. Since the multiplication $F_{2} \otimes F_{2} \rightarrow R$ is a perfect pairing there is a basis $f_{1}^{\prime}, \ldots, f_{m}^{\prime}$ for $F_{2}$ such that $f_{i} f_{j}^{\prime}=-\delta_{i j}$. If $f_{i}^{\prime}=\sum s_{i j} f_{j}$, then the change of basis matrix $s=\left(s_{i j}\right)$ is clearly invertible and symmetric. Both (2) and (4) follow by direct computation.

Proposition 3.5. Let $S$ be a commutative ring and let

$$
\begin{aligned}
& 0 \rightarrow S \stackrel{\mathbf{b}^{t}}{\rightarrow} \quad S^{3} \stackrel{B}{\rightarrow} S^{3} \stackrel{\text { b }}{\rightarrow} S \\
& 0 \rightarrow S \stackrel{\mathbf{a}^{\prime}}{\rightarrow} \quad S^{n} \stackrel{s A^{t}}{\rightarrow} S^{2 n-2} \stackrel{A}{\rightarrow} S^{n} \stackrel{\text { a }}{\rightarrow} S
\end{aligned}
$$

be a commutative diagram with exact rows, where $s$ is an invertible symmetric matrix, $B$ is alternating, $\mathbf{c} X=0$, and $M^{t} s^{-1} M=0$. Let $T=S[v]$ where $v$ is an indeterminate, and let $\mathbf{F}$ be

$$
0 \rightarrow T \stackrel{d_{4}}{\rightarrow} T^{n+3} \stackrel{d_{3}}{\rightarrow} T^{2 n+4} \stackrel{d_{2}}{\rightarrow} T^{n+3} \stackrel{d_{1}}{\rightarrow} T
$$


with $d_{1}=[\mathbf{b}, \mathbf{c}+v \mathbf{a}], d_{4}=d_{1}^{t}$,

$$
d_{2}=\left[\begin{array}{ccc}
v I_{3} & B & M^{t} s^{-1} \\
-X & 0 & -A
\end{array}\right] \text { and } d_{3}=\left[\begin{array}{cc|c}
0 & I & \\
& & 0 \\
I & 0 & \\
\hline & 0 & s
\end{array}\right] d_{2}^{t} .
$$

Then $\mathbf{F}$ and $\mathbf{F} \otimes_{T} S \llbracket v \rrbracket$ are both acyclic.

Proof. Apply Theorem 1.3 of [23] to the commutative diagram

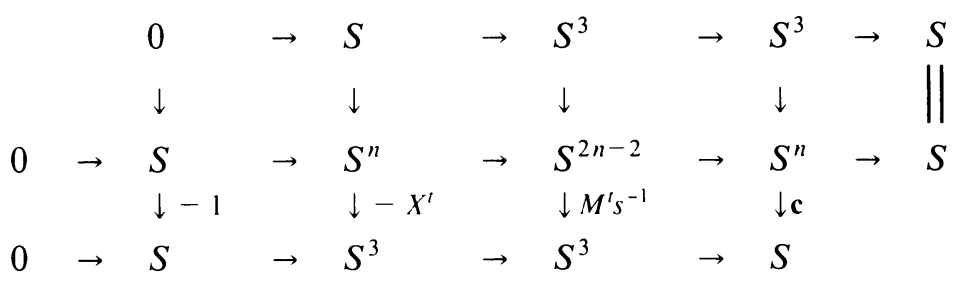

where the remaining maps are indicated in (3.3). Since all the vertical composition maps are zero, we can take all $h_{i}=0$ in the cited theorem. The complex $\mathbf{F} \otimes_{S[v]} S \llbracket v \rrbracket$ is acyclic because $\mathbf{F}$ is, and the inclusion $S[v] \rightarrow S \llbracket v \rrbracket$ is flat.

We now record the minimal resolution of $A(l+1, \tau)$ in terms of the minimal resolution of $A(l, \tau)$. Let $\mathbf{F}$ be an associative DG algebra which is a minimal resolution of $A(l, \tau)$ by free $R$-modules for $R=R(l, \tau)$. By Lemma 3.4 we can select bases so that there is a symmetric invertible matrix $s$, and $\mathbf{F}$ has the form exhibited in the lower row of (3.3). Let $X$ be an $n \times 3$ matrix of indeterminates, $\mathbf{b}=\mathbf{a} X$, $S=R \llbracket X \rrbracket$, and $\mathbf{K}$ be the Koszul resolution of $S /(\mathbf{b})$, with bases chosen so that

$$
B=\left[\begin{array}{ccc}
0 & b_{3} & -b_{2} \\
-b_{3} & 0 & b_{1} \\
b_{2} & -b_{1} & 0
\end{array}\right]
$$

If $\alpha: \mathbf{K} \otimes_{R} S$ is a map of associative DG algebras represented by the matrices $X, M$ and $\mathbf{c}^{t}$, then since $K_{4}=0$ and the multiplication on F satisfies (3) and (4) of Lemma 3.4, we see that $\mathbf{c} X=0$ and $M^{t} s^{-1} M=0$. It follows that Proposition 3.5 gives a resolution of $A(l+1, \tau)$ by free $S \llbracket v \rrbracket=R(l+1, \tau)$-modules. Each entry of $M$ is in $\left(x_{i j}\right)^{2}$ and each entry of $\mathbf{c}$ is in $\left(x_{i j}\right)^{3}$. Hence all the matrices involved have all entries in the maximal ideal of $R(l+1, \tau)$, and the resolution is minimal.

The identical construction applied to $A(0, \tau)$ with $X$ as in (3.1) gives a resolution of $B(\tau)$. The resolution is not minimal because one entry of $d_{2}$ and one entry of $d_{3}$ are units. By induction on $l$ we have established

Corollary 3.6. (1) If $A(l, \tau)=R(l, \tau) / J$, then $\mu(J)=3 l+\tau+1$.

(2) If $B(\tau)=R(\tau) / U$, then $\mu(J)=3+\tau$.

4. Preservation of rigidity under linkage. We show that rigidity is preserved under "general" linkage (Theorem 4.2), and consequently that the "most general" double link of a rigid Gorenstein algebra is still rigid (Remark 4.3.). Note this is not the general double link of Definition 2.5. Our goal, achieved at the end of $\S 5$, is to show that the algebras $A(l, \tau)$ and $B(\tau)$ are rigid. The following result shows that 
infinitesimal deformation passes over a linkage. We fix an algebraically closed field $k$. Let $k^{\prime}=k[\varepsilon] /\left(\varepsilon^{2}\right)$ be the ring of dual numbers, and for a $k$-algebra $R$, let $R^{\prime}=R \otimes_{k} k^{\prime}$.

LemMa 4.1. Let $R$ be a Gorenstein local $k$-algebra, $J$ a perfect grade $g$ ideal of $R$, and $\mathbf{z} \subseteq J$ a length $g$ regular sequence. If $I$ is linked to $J$ by $\mathbf{z}$ and $R^{\prime} / J^{\prime}$ is a deformation of $R / J$ to $k^{\prime}$, then there is a length $g$ regular sequence $\mathbf{z}^{\prime}$ in $R^{\prime}$ and a deformation $R^{\prime} / I^{\prime}$ of $R / I$ such that $I^{\prime}$ is linked to $J^{\prime}$ by $\mathbf{z}^{\prime}$ and $R^{\prime} /\left(\mathbf{z}^{\prime}\right)$ is a deformation of $R /(\mathbf{z})$.

Proof. Let $\mathbf{d}$ be a vector whose entries minimally generate $J$ and let $\alpha_{1}$ be a matrix with $\mathbf{z}=\mathbf{d} \alpha_{1}$. Let $\mathbf{G}$ and $\mathbf{F}$ be the minimal resolutions of $R /(\mathbf{z})$ and $R / J$ (respectively) by free $R$-modules. Since $I$ is linked to $J$ by $\mathbf{z}$, we have $I=(\mathbf{z}): J$ and by Theorem 1.1 a minimal resolution of $R / I$ is obtained by taking the mapping cone $\mathbf{M}$ of the dual of a comparison map:

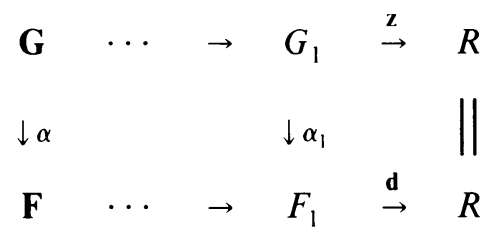

Since $R^{\prime} / J^{\prime}$ is a deformation of $R / J$, there is a row vector $\xi$ with entries in $R$ so that $J^{\prime}$ is generated by $\mathbf{d}+\varepsilon \xi$, and there is a resolution $\mathbf{F}^{\prime}$ of $R^{\prime} / J^{\prime}$ by free $R^{\prime}$-modules with $\mathbf{F}^{\prime} \otimes_{k^{\prime}} k=\mathbf{F}$. Let $\mathbf{z}^{\prime}=(\mathbf{d}+\varepsilon \xi) \alpha_{1}$, and let $I^{\prime}=\left(\mathbf{z}^{\prime}\right): J^{\prime}$. It is clear that $I^{\prime}$ is linked to $J^{\prime}$ by $\mathbf{z}^{\prime}$ and that $R^{\prime} /\left(\mathbf{z}^{\prime}\right)$ is a deformation of $R /(\mathbf{z})$. We must show that $R^{\prime} / I^{\prime}$ is a deformation of $R / I$. Let $\mathbf{G}^{\prime}$ be a resolution of $R^{\prime} /\left(\mathbf{z}^{\prime}\right)$ by free $R^{\prime}$-modules which lifts the resolution $\mathbf{G}$ of the complete intersection $R /(\mathbf{z})$. Let $\alpha^{\prime}$ : $\mathbf{G}^{\prime} \rightarrow \mathbf{F}^{\prime}$

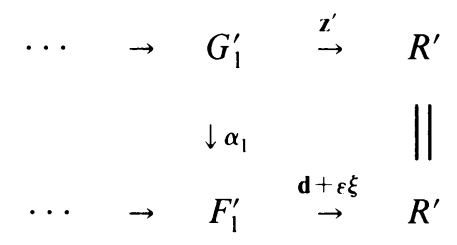

be a map of resolutions which lifts $\alpha: \mathbf{G} \rightarrow \mathbf{F}$ where $G_{i}^{\prime}=G_{i} \otimes_{R} R^{\prime}$ and $F_{i}^{\prime}=F_{i} \otimes_{R} R^{\prime}$. The mapping cone $\mathbf{M}^{\prime}$ of the dual of $\alpha^{\prime}$ is a resolution of $R^{\prime} / I^{\prime}$ and $\mathbf{M}^{\prime} \otimes_{k^{\prime}} k=\mathbf{M}$. It follows that $R^{\prime} / I^{\prime}$ is a deformation of $R / I$.

THEOREM 4.2. Let $A$ be a collection of indeterminates and a be a $1 \times n$ matrix which generates a perfect grade $g$ ideal of $S=k \llbracket A \rrbracket$. Suppose $S /($ a) is a rigid $k$-algebra. Let $X$ be the $n \times g$ matrix

$$
X=\left[\begin{array}{l|l}
I_{t} & 0 \\
\hline Y
\end{array}\right]
$$

where $Y$ is an $(n-t) \times g$ matrix of indeterminates and $0 \leqslant t \leqslant g$. Assume that $\mathbf{z}=\mathbf{a} X$ is a regular sequence in $R=S \llbracket Y \rrbracket$. If $J$ is linked to $I=(\mathbf{a}) R$ by $(\mathbf{z})$ in $R$, then $R / J$ is a rigid $k$-algebra. 
Proof. Suppose $R^{\prime} / J^{\prime}$ is a first order deformation of $R / J$ to $k^{\prime}$. By Lemma 4.1 there is a regular sequence $\mathbf{z}^{\prime}$ and a deformation $R^{\prime} / I^{\prime}$ of $R / I$ such that $I^{\prime}$ is linked to $J^{\prime}$ over $\left(\mathbf{z}^{\prime}\right)$. Since $S /(\mathbf{a})$ is rigid, so is $R / I=(S /(\mathbf{a})) \llbracket Y \rrbracket$ and hence $R^{\prime} / I^{\prime}$ must represent a trivial deformation; that is, there is an isomorphism of $k^{\prime}$-algebras $\theta$ : $R / I \otimes_{k} k^{\prime} \rightarrow R^{\prime} / I^{\prime}$ that is the identity on the fiber $\varepsilon=0$. We view $\theta$ as a $k^{\prime}$-automorphism of $R^{\prime}=k^{\prime} \llbracket A, Y \rrbracket$ such that $\theta\left((\mathbf{a}) R^{\prime}\right)=I^{\prime}$ and $\left.\theta\right|_{\varepsilon=0}$ is the identity on $k \llbracket A, Y \rrbracket$ (see Proposition C). Therefore, if we ignore $\theta(Y)$, there is a $k^{\prime}$-algebra map $\theta: S^{\prime} \rightarrow R^{\prime}$ which has the properties:

(1) for each $s \in S$, there is an $r \in R$ with $\theta(s)=s+\varepsilon r$;

(2) the image $\mathbf{a}^{\prime}=\mathbf{a}+\varepsilon \xi_{2}=\theta(\mathbf{a})$ of the vector a generates the ideal $I^{\prime}$. Let

$$
S^{m} \stackrel{d_{2}}{\rightarrow} S^{n} \stackrel{\mathbf{a}}{\rightarrow} S
$$

be a presentation. Since $\left(\mathbf{z}^{\prime}\right) \subseteq\left(\mathbf{a}^{\prime}\right)$, there are matrices $M$ and $M^{\prime}$ with entries in $R$ such that $\mathbf{z}^{\prime}=\mathbf{a}^{\prime}\left(M+\varepsilon M^{\prime}\right)$. Since $\mathbf{z}^{\prime}=\mathbf{z}+\varepsilon \xi_{1}$ and $\mathbf{a}^{\prime}=\mathbf{a}+\varepsilon \xi_{2}$, we see that a $(M-X)=0$; thus $M$ differs from $X$ by $d_{2} N$, for some matrix $N$. On the other hand, $\xi_{2}$ represents a deformation of $R / I$; hence $\xi_{2} d_{2} N=\mathbf{a} N^{\prime}$ for some $N^{\prime}$, and we may modify $M^{\prime}$ in order to assume $M=X$. Thus we have $\mathbf{z}^{\prime}=\mathbf{a}^{\prime}\left(X+\varepsilon M^{\prime}\right)$ where $M^{\prime}$ has entries in $R$; and there is an invertible matrix $U$ with entries in $R^{\prime}$ such that

$$
\mathbf{z}^{\prime} U=\mathbf{a}^{\prime}\left[\begin{array}{l|l}
I & 0 \\
\hline Y+\varepsilon Y^{\prime}
\end{array}\right]
$$

for some matrix $Y^{\prime}$. Certainly $\left(\mathbf{z}^{\prime}\right)=\left(\mathbf{z}^{\prime} U\right)$ as ideals (but not as row vectors). The extension of $\theta$ to $R^{\prime} \rightarrow R^{\prime}$ which sends $y_{i j}$ to $y_{i j}+\varepsilon y_{i j}^{\prime}$ also sends (a) to $\left(\mathbf{a}^{\prime}\right),(\mathbf{z})$ to $\left(\mathbf{z}^{\prime}\right)$ and $J=\left(\mathbf{z}:\right.$ a) to $\left(\mathbf{z}^{\prime}: \mathbf{a}^{\prime}\right)=J^{\prime}$. Therefore, $R^{\prime} / J^{\prime}$ is a trivial deformation of $R / J$, and we have shown that $R / J$ is rigid.

As an immediate application of the theorem, we recover the rigidity of "special" almost complete intersections proved by Herzog [13, Satz 3.4d] and De Carli and Gabelli [9]. In this context $n=g, \mathbf{a}=\left[A_{1}, \ldots, A_{g}\right], t=0$, and $J$ is the almost complete intersection (a $X, \operatorname{det} X)$.

RemarK 4.3. Take $S, \mathbf{a}, Y, X, \mathbf{z}$ and $J$ as in Theorem 4.2. Assume also that (a) is a Gorenstein ideal. Then by Theorem 1.2 we may assume $J=(w, \mathbf{z})$ for some $w$ in $S \llbracket Y \rrbracket$. If $v_{1}, \ldots, v_{g}$ are new indeterminates and

$$
\mathbf{e}=[w, \mathbf{z}]\left[\frac{I_{g}}{v_{1} \cdots v_{g}}\right]
$$

is a regular sequence, then Theorems 4.2 and 1.2 guarantee that $R / K$ is a rigid Gorenstein $k$-algebra, where $K=(\mathbf{e}): J$ and $R=S \llbracket Y, v_{1}, \ldots, v_{g} \rrbracket$.

In the next section we show that with one additional hypothesis, most of the indeterminates $v_{i}$ and some of the indeterminates $y_{i j}$ are redundant.

5. The algebras $A(l, \tau)$ and $B(\tau)$ are rigid. Throughout this section $A$ denotes a finite collection of indeterminates, $k$ is an algebraically closed field, $S=k \llbracket A \rrbracket$, and a is a $1 \times n$ matrix that minimally generates a grade $g$ Gorenstein ideal in $S$. We 
assume that $S /($ a) is rigid. Let $X$ be an $n \times(g-1)$ matrix of form

$$
X=\left[\begin{array}{l|l}
I_{t} & 0 \\
\hline Y
\end{array}\right]
$$

where $0 \leqslant t \leqslant g-1$ and $Y$ is an $(n-t) \times(g-1)$ matrix of indeterminates. Assume that $\mathbf{b}=\mathbf{a} X$ is a regular sequence in $R=S \llbracket X \rrbracket$. (If $t=0$ this is true by Proposition A.) Denote the minimal free resolutions of $R /(\mathbf{a})$ and $R /(\mathbf{b})$ by $\mathbf{F}$ and $\mathbf{G}$, respectively. Define a comparison map $\alpha: \mathbf{G} \rightarrow \mathbf{F}$ as described just before (2.7). The matrix $\mathbf{c}$ of (2.7) completes diagram (2.2). Let $I=\left(\mathbf{b}, \mathbf{c}+v_{g} \mathbf{a}\right)$, where $v_{g}$ is an indeterminate.

THEOREM 5.1. With the notation and hypotheses made above, the algebra $R \llbracket v_{g} \rrbracket / I$ is rigid.

Proof. In Theorem 4.2 the matrix $X$ has an extra column; in the case $t=0$ this makes a $X$ the "most general" regular sequence of length $g$ in (a). The present construction of $I$ lacks these extra variables, so we simply adjoin $\left[0, \ldots, 0, Z_{1}, \ldots, Z_{n-t}\right]^{t}$ to $X$ as the last column, and we set $y=\sum_{i=1}^{n-t} a_{t+i} Z_{i}$. By hypothesis $\mathbf{b}$ is a regular sequence in $(\mathbf{a})$, so $\operatorname{grade}(\mathbf{a}) /(\mathbf{b})=1$. By Proposition A, we have a regular sequence $\mathbf{b}, \sum_{i=1}^{n} a_{i} T_{i}$, where the $T_{i}$ are indeterminates. Observe that

$$
\sum_{j=1}^{n} a_{j} T_{j}-\sum_{j=1}^{t} b_{j} T_{j}=\sum_{i=1}^{n-t} a_{i+t}\left(T_{i+t}-\sum_{j=1}^{t} y_{i j} T_{j}\right)
$$

is a regular element in (a) on $R \llbracket T \rrbracket /(\mathbf{b})$. After a change of variables it is clear that $\mathbf{b}$, $y$ is a regular sequence in (a) on $R \llbracket Z \rrbracket$. By Theorem 4.2 we conclude that $R \llbracket Z \rrbracket / J$ is rigid, where $J=(\mathbf{b}, y)$ : (a). If we apply $-\bigotimes_{R} R \llbracket Z \rrbracket$ to diagram (2.2), then by Theorem 2.1 there is an element $w$ in $R \llbracket Z \rrbracket$ such that $I R \llbracket Z, v_{g} \rrbracket$ has the form (b, $\left.w+v_{g} y\right): y$ in $R \llbracket Z, v_{g} \rrbracket$. In fact, $J=(\mathbf{b}, y, w)$ and $w=\alpha_{g}$ as in (2.3). In that diagram the map $\alpha$ from $\mathbf{K}=\mathbf{K}(\mathbf{b}, y)$ to $\mathbf{F}$ is the natural extension of our original $\operatorname{map} \alpha: \mathbf{G} \rightarrow \mathbf{F}$. In particular,

$$
w=\sum_{1 \leqslant i_{1}<\cdots<i_{g} \leqslant n} e_{i_{1}}\left(e_{i_{2}}\left(\cdots\left(e_{i_{g}}\right) \cdots\right)\right) M\left(i_{1}, \ldots, i_{g} ; 1, \ldots, g\right)
$$

where

$$
M=\left[\begin{array}{l|l|l}
I_{t} & 0 & 0 \\
\hline Y & Z
\end{array}\right]
$$

Now let $v_{1}, \ldots, v_{g-1}$ be new indeterminates and

$$
\mathbf{e}=[\mathbf{b}, w, y]\left[\begin{array}{c}
I_{g} \\
v_{1} \cdots v_{g}
\end{array}\right] .
$$

If $\mathbf{e}^{\prime}=\left[b_{1}+v_{1} y, \ldots, b_{g-1}+v_{g-1} y\right]$, then $\left[\mathbf{e}^{\prime}, y\right]$ generates an ideal of height $g$ in a local ring and hence is a regular sequence. The polynomial $w+v_{g} y$ is regular on $\left(R \llbracket Z, v_{1}, \ldots, v_{g-1} \rrbracket /\left(\mathbf{e}^{\prime}\right)\right)\left[v_{g}\right]$ and by flatness still regular on $R \llbracket Z, V \rrbracket /\left(\mathbf{e}^{\prime}\right)$. (Here $V=\left\{v_{1}, \ldots, v_{g}\right\}$.) Since $\mathrm{e}$ is thus a regular sequence, Theorem 4.2 implies that $R \llbracket Z, V \rrbracket / I^{\prime}$ is rigid, where $I^{\prime}=(\mathbf{e}): J$. 
To show that $B=R \llbracket v_{q} \rrbracket / I$ is rigid, it will suffice to show that

$$
B \llbracket v_{1}, \ldots, v_{g-1}, Z \rrbracket \simeq R \llbracket Z, V \rrbracket / I^{\prime} .
$$

Define an $S \llbracket Z, V \rrbracket$-algebra automorphism $\theta$ of $R \llbracket Z, V \rrbracket$ by setting $\theta\left(y_{i j}\right)=y_{i j}+$ $Z_{i} v_{j}$. Observe that $\theta(y)=y$ and $\theta\left(b_{i}\right)=b_{i}+y v_{i}=e_{i}$ for $1 \leqslant i \leqslant g-1$. Also $\theta(M)$ differs from $M$ only by column operations; hence $\theta(w)=w$. Consequently,

$$
\theta(I)=\theta\left[\left(\mathbf{b}, w+v_{g} y\right): y\right]=(\mathbf{e}): y=I^{\prime} .
$$

COROLlaRy 5.2. The general double link of a rigid Gorenstein algebra is rigid. In particular each $A(l, \tau)$ is rigid.

Proof. The first assertion is simply Theorem 5.1 with $t=0$. The rigidity of $A(l, \tau)$ follows from this fact together with Proposition 3.1(3).

COROllary 5.3. Each algebra $B(\tau)$ is rigid.

Proof. Since $B^{\prime}(\tau)=B(\tau) \llbracket x_{21}, \ldots, x_{\tau 1} \rrbracket$ (see Example 3.2) it suffices to show that $B^{\prime}(\tau)$ is rigid. Since $A(0, \tau)$ is rigid, and $X$ as in (3.2) has the requisite form for application of Theorem 5.1, we conclude that $B^{\prime}(\tau)$ is rigid.

6. Regularity conditions. In this section we show that the algebras $A(l, \tau)$ and $B(\tau)$ are regular in codimension six. Since these algebras are local and Gorenstein, they are consequently normal domains. Our technique is to show that in the presence of rigidity the complete intersection locus and the regular locus coincide. The following condition on the local number of generators of an ideal $J$ in a ring $R$ is central to the discussion.

$\left(\mathrm{LG}_{i}\right) \quad \mu\left(J_{\mathfrak{p}}\right) \leqslant \max \{g$, ht $\mathfrak{p}-i\} \quad$ for all primes $\mathfrak{p}$ containing $J$.

If $J$ is a grade $g$ ideal in a Cohen-Macaulay ring $R$ and $J$ satisfies $\left(\mathrm{LG}_{i}\right)$, then $A=R / J$ is a complete intersection at all primes of height at most $i$. We indicate how the property $\left(\mathrm{LG}_{i}\right)$ for $i \leqslant 4$ and a somewhat weaker one for $i \leqslant 6$ pass across various kinds of double links, including the general double link. The section concludes with several results concerning graded algebras associated to $R$ and $J$ in the context that $A=R / J$ is in the linkage class of a complete intersection. As usual we fix $g \geqslant 3$ and $k$ an algebraically closed field.

The following theorem is of interest in a wider context than this section. It is known (see Ulrich [29, §4]) that if $R=S / I$ is reduced (with $S$ regular local as below), then $R_{\mathfrak{p}}$ is regular if and only if $R_{\mathfrak{p}}$ is a rigid complete intersection. We remove the hypothesis that $R$ be reduced, but we impose the additional assumption that $R$ be Gorenstein. The proof-but not the statement-of this result is contained in $[\mathbf{2 0}, \S 10]$, where, however, the unnecessarily strong hypothesis of an associative DG structure is imposed. For the sake of completeness we reproduce the brief argument.

THEOREM 6.1. Let $S=k\left[X_{1}, \ldots, X_{m}\right]_{(X)}$ or $k \llbracket X_{1}, \ldots, X_{m} \rrbracket$ and let $I$ be a grade $g$ Gorenstein ideal in $S$. Assume that $S / I$ is a rigid k-algebra. If $P$ is a prime ideal containing I such that $(S / I)_{P}$ is a complete intersection, then $(S / I)_{P}$ is regular. 
Proof. By [4] any minimal free resolution of $S / I$

$$
\mathbf{F}: 0 \rightarrow S \rightarrow F_{g-1} \rightarrow \cdots \stackrel{d_{2}}{\rightarrow} F_{1} \stackrel{d_{1}}{\rightarrow} S
$$

may be given a homotopy associative DG algebra structure. We may assume that $F_{1}$ has a free basis $\left\{x_{1}, \ldots, x_{n}\right\}$ such that $I_{P}=\left(d x_{1}, \ldots, d x_{g}\right)_{P}$. We claim that the element $x_{1}\left(x_{2}\left(\cdots\left(x_{g}\right)\right)\right)$ of $F_{g}=S$ is not in $P$. By passing to $S_{P}$ we have $\mathbf{F}=\mathbf{K} \oplus \mathbf{H}$, where $\mathbf{K}=\Lambda\left(\Sigma S x_{i}\right)$ is the Koszul resolution. The DG algebra on $\mathbf{F}$ may be restricted to give a DG algebra on $\mathbf{K}$; both this algebra and the exterior algebra induce the same algebra on $\mathbf{K} \otimes_{S}(S / P)_{P}$. Thus $x_{1} \wedge \cdots \wedge x_{g} \equiv x_{1}\left(x_{2} \cdots\left(x_{g}\right) \cdots\right)$ modulo $P$. The exterior product is a unit in $K_{g}=S_{p}$; so therefore is $x_{1}\left(x_{2}\left(\cdots\left(x_{g}\right)\right)\right)$.

By the homotopy associativity, any other association of the elements $x_{1}, \ldots, x_{g}$ differs from $x_{1}\left(x_{2}\left(\cdots\left(x_{g}\right)\right)\right)$ by an element of $I$, and thus is not in $P$. Let $f_{i}$ : $F_{1} \rightarrow F_{g}=S$ be the map which sends $x$ to

$$
x\left(x_{1}\left(x_{2}\left(\cdots\left(\hat{x}_{i}\left(\cdots\left(x_{g}\right)\right)\right)\right)\right)\right) .
$$

The differential property shows that the image of $f_{i} d_{2}$ is in $I$ and thus $f_{i}$ represents a first order deformation of $S / I$. Rigidity implies that for each $i$ the image of $f_{i}^{t}$ modulo $I$ is in the column space of $\mathcal{G}$, the Jacobian of $d_{1}$. But we have already established that in $(S / P)_{P}$

$$
f_{i}\left(x_{i}\right)=\left[0, \ldots, u_{i}, 0, \ldots, 0, *, \ldots, *\right]
$$

with $u_{i}$ a unit in the $i$ th component for $1 \leqslant i \leqslant g$. Thus $\operatorname{rank}(q) \geqslant g$ at $P$ and $(S / I)_{P}$ is regular by $[26, \S 29]$.

COROllary 6.2. Let $K$ be a grade $g$ Gorenstein ideal in $S=k\left[X_{1}, \ldots, X_{m}\right]_{(X)}$ or $k \llbracket X_{1}, \ldots, X_{m} \rrbracket$. If $S / K$ is rigid and $K$ satisfies $\left(\mathrm{LG}_{1}\right)$, the $S / K$ is a normal domain.

Proof. The condition $S_{2}$ holds since $S / K$ is Gorenstein. The condition $R_{1}$ follows from Theorem 6.1. Hence $S / K$ is a normal ring, and it is a domain since it is local.

The following result will enable us to pass the condition $\left(\mathrm{LG}_{4}\right)$ from $A(l, \tau)$ to $A(l+1, \tau)$.

THEOREM 6.3. Let a be a $1 \times n$ vector which minimally generates a grade $g$ Gorenstein ideal $J$ in a Gorenstein local ring $R$. Let $X$ be an $n \times(g-1)$ matrix of indeterminates and $\mathbf{c}$ be $a 1 \times n$ vector as in diagram (2.2). Let $K=(\mathbf{a} X, \mathbf{c}+v \mathbf{a})$ be the grade $g$ Gorenstein ideal doubly linked to (a) in $S=R \llbracket X, v \rrbracket$, as in Theorem 2.1. Let $i$ be an integer, $0 \leqslant i \leqslant 4$, and assume that $J$ satisfies $\left(\mathrm{LG}_{i}\right)$ in $R$. Then $K$ satisfies $\left(\mathrm{LG}_{i}\right)$ in $S$.

Proof. By Proposition A we know $\mathbf{b}=\mathbf{a} X$ is a regular sequence of length $g-1$ in $R \llbracket X \rrbracket$. We can choose an element $y$ in $J$ so that $y$ is regular on $R \llbracket X \rrbracket /(b)$. Let $I=(\mathbf{b}, y): J$. By the proof of Theorem 2.1, particularly (2.4), there exists an element $w$ in $R \llbracket X \rrbracket$ such that $I=(\mathbf{b}, y, w), K=(\mathbf{b}, w+v y): I$, and

$$
w a_{i} \equiv y c_{i} \bmod (\mathbf{b})
$$


for $0 \leqslant i \leqslant n$. Let $P$ be a prime in $S$ that contains $K$. If $P$ does not contain $I$, then $K_{P}=(\mathbf{b}, w+v y)_{P}$ is a complete intersection. We assume $P$ contains $I$ but does not contain $J$. Then $I_{P}=(\mathbf{b}, y)_{P}$ is a complete intersection. Since $K_{P}$ is linked to $I_{P}$ in $S_{P}$, we know $\mu\left(K_{P}\right) \leqslant g+1$. But $K_{P}$ is a codimension $g$ Gorenstein ideal, so $\mu\left(K_{P}\right)=g$ by Kunz [19]. We assume, henceforth, that $P \supset I+J+K$, and we let $\mathfrak{p}=P \cap R$, noting that $\mathfrak{p} \supset J$. Let $t=\mu\left(J_{\mathfrak{p}}\right)$. By $\left(\mathrm{LG}_{i}\right)$ we have

$$
g \leqslant t \leqslant \max \{g, \text { ht } \mathfrak{p}-i\} \leqslant \max \{g, \text { ht } P-i\} .
$$

After possibly reindexing and by introducing a common denominator, we may assume there is an element $\alpha \in R \backslash \mathfrak{p}$ such that $\mathbf{a}^{\prime}=\left[a_{1}, \ldots, a_{t}\right]$ generates $J_{\mathfrak{p}}$ and $\mathbf{a}=\mathbf{a}^{\prime}\left[I_{t} \mid \alpha^{-1} U\right]$ for some $t \times(n-t)$ matrix $U$ with entries in $R$. Let $\mathbf{c}^{\prime}=\left[c_{1}, \ldots, c_{t}\right]$. By (6.1)

$$
\begin{aligned}
y \alpha\left[c_{t+1}+v a_{t+1}, \ldots, c_{n}+v a_{n}\right] & \equiv(w+v y) \alpha\left[a_{t+1}, \ldots, a_{n}\right] \\
& =(w+v y) \mathbf{a}^{\prime} U \equiv y\left(\mathbf{c}^{\prime}+v \mathbf{a}^{\prime}\right) U
\end{aligned}
$$

modulo (b). Since (b, $y$ ) is a regular sequence we conclude $K_{\alpha}=\left(\mathbf{b}, \mathbf{c}^{\prime}+v \mathbf{a}^{\prime}\right)_{\alpha}$ and hence

$$
\mu\left(K_{P}\right) \leqslant g-1+t .
$$

Let $X^{\prime}$ be the $t \times(g-1)$ matrix $\left[\alpha I_{t} \mid U\right] X$. Then

$$
\alpha \mathbf{b}=\alpha \mathbf{a} X=\mathbf{a}^{\prime} X^{\prime} \text {. }
$$

Let $I_{r}\left(X^{\prime}\right)$ be the ideal in $S$ generated by the $r \times r$ minors of $X^{\prime}$.

Claim 1. If $P$ contains $I_{r}\left(X^{\prime}\right)$, then ht $P \geqslant$ ht $\mathfrak{p}+(t-r+1)(g-r)$.

View $I_{r}\left(X^{\prime}\right)$ as an ideal in $R_{\alpha}[X]$. Observe that the entries $X_{i j}^{\prime}$ of $X^{\prime}$ are algebraically independent over $R_{\alpha} / \mathfrak{p}$. In fact, $\left(R_{\alpha} / \mathfrak{p}\right)\left[X_{i j}^{\prime}, X_{l k}\right]=\left(R_{\alpha} / \mathfrak{p}\right)[X]$, where $1 \leqslant j, k \leqslant g-1,1 \leqslant i \leqslant t$, and $t+1 \leqslant l \leqslant n$. By the theory of determinantal ideals [17, Theorem 1, Corollary 4], $\left(I_{r}\left(X^{\prime}\right)+\mathfrak{p}\right) / \mathfrak{p}$ is a prime ideal of height $(t-r+1)$ $\times(g-r)$ in $\left(R_{\alpha} / \mathfrak{p}\right)[X]$, so ht $\mathfrak{p}+(t-r+1)(g-r) \leqslant \operatorname{ht}\left(I_{r}\left(X^{\prime}\right)+\mathfrak{p}\right)$. The map $R[X]_{\alpha} \rightarrow R \llbracket X, v \rrbracket_{\alpha}=S$ is flat, so ht $P \geqslant \operatorname{ht}\left(P \cap R[X]_{\alpha}\right) \geqslant \operatorname{ht}\left(I_{r}\left(X^{\prime}\right)+\mathfrak{p}\right)$, which establishes the claim.

Claim 2. If $P$ does not contain $I_{r}\left(X^{\prime}\right)$, then $\mu\left(K_{P}\right) \leqslant g-1+t-r$.

Some $r \times r$ minor of $X^{\prime}$ lies outside of $P$. After possibly reindexing, we may assume the upper left-hand minor of $X^{\prime}$ is not in $P$. If we multiply (6.4) by $w+v y$ and use (6.1) together with the fact that $(\mathbf{b}, y)$ is a regular sequence, then we see that each entry of $\left(\mathbf{c}^{\prime}+v \mathbf{a}^{\prime}\right) X^{\prime}$ is in $(\mathbf{b})_{P}$. Hence we can solve for $c_{1}^{\prime}+v a_{1}^{\prime}, \ldots, c_{r}^{\prime}+v a_{r}^{\prime}$ (in $S_{P}$ ) in terms of $c_{r+1}^{\prime}+v a_{r+1}^{\prime}, \ldots, c_{t}^{\prime}+v a_{t}^{\prime}$, and $\mathbf{b}$. It follows that

$$
K_{P}=\left(\mathbf{b}, c_{r+1}^{\prime}+v a_{r+1}^{\prime}, \ldots, c_{t}^{\prime}+v a_{t}^{\prime}\right)_{P}
$$

and $\mu\left(K_{P}\right) \leqslant g-1+t-r$, as claimed.

Estimation of $\mu\left(K_{P}\right)$ now boils down to a case analysis. There are three possibilities:

(i) ht $\mathfrak{p}-i \leqslant$ ht $P-i \leqslant g=t$.

(ii) ht $\mathfrak{p}-i \leqslant g=t<$ ht $P-i$.

(iii) $g<t \leqslant$ ht $\mathfrak{p}-i \leqslant$ ht $P-i$. 
In each case one finds an index $r$ such that $P$ contains $I_{r+1}\left(X^{\prime}\right)$ but not $I_{r}\left(X^{\prime}\right)$ and applies the claims established above. Since $X^{\prime}$ is $t \times(g-1)$, such an index must exist in the range $0 \leqslant r \leqslant g-1$. To see why the restriction $i \leqslant 4$ is needed, consider case (ii) with $1 \leqslant r \leqslant g-3$. From the two claims we obtain ht $P \geqslant$ ht $p+$ $(g-r)(g-r-1)$ and

$$
\begin{aligned}
\mu\left(K_{P}\right) & \leqslant 2 g-1-r \leqslant 2 g-1-r+(g-r)(g-r-2)-3 \\
& =g+(g-r)(g-r-1)-4 \\
& \leqslant \text { ht } p+(g-r)(g-r-1)-4 \leqslant \text { ht } P-i .
\end{aligned}
$$

The other arguments are similar. In a few cases one obtains only $\mu\left(K_{P}\right) \leqslant g+1$, but by Kunz's theorem this implies $\mu\left(K_{P}\right)=g$.

As noted above if $K$ satisfies $\left(\mathrm{LG}_{i}\right)$ then $K_{P}$ is a complete intersection at primes $P$ such that ht $P \leqslant g+i$. This formally weaker property (which, however, is all we need for application of Theorem 6.1) is preserved over double link as in Theorem 6.3 up through $i \leqslant 6$. In fact, we can show this for the more general matrix $X$ that appears in $\$ 5$ and the construction of $B(\tau)$.

THEOREM 6.4. Let a be a $1 \times n$ vector which minimally generates a grade $g$ Gorenstein ideal $J$ in a Gorenstein local ring $R$. Let $Y$ be an $(n-t) \times(g-1)$ matrix of indeterminates, $0 \leqslant t \leqslant g-1$,

$$
X=\left[\begin{array}{l|l}
I_{t} & 0 \\
\hline Y
\end{array}\right]
$$

and $\mathbf{c}$ be a vector as in (2.2). Assume that $\mathbf{b}=\mathbf{a} X$ is a regular sequence. Let $K=(\mathbf{b}, \mathbf{c}+v \mathbf{a})$ in $S=R \llbracket Y, v \rrbracket$, and $i$ be an integer with $0 \leqslant i \leqslant 6$. If $J_{\mathfrak{p}}$ is a complete intersection for all primes $\mathfrak{p}$ of $R$ containing $J$ with ht $\mathfrak{p} \leqslant g+i$, then $K_{P}$ is a complete intersection for all primes $P$ of $S$ containing $K$ with ht $P \leqslant g+i$.

Proof. Suppose $P$ is a prime of $S$ containing $K$ with ht $P \leqslant g+i$. As in the proof of Theorem 6.3 we need only consider the case that $\mathfrak{p}=P \cap R$ contains $J$. Then ht $\mathfrak{p} \leqslant g+i$ and $J_{\mathfrak{p}}$ is generated by $g$ of the elements of a. Let us form a minimal generating set by taking as many possible, say $l$, from $\left\{a_{1}, \ldots, a_{t}\right\}$ and the other $m=g-l$ from $\left\{a_{t+1}, \ldots, a_{n}\right\}$. After reindexing we have $J_{\mathfrak{p}}=\left(\mathbf{a}^{\prime}\right)_{\mathfrak{p}}$ where $a^{\prime}=$ $\left[a_{1}, \ldots, a_{l}, a_{t+1}, \ldots, a_{t+m}\right]$ and $a_{j} \in\left(\left(a_{1}, \ldots, a_{l}\right)+\mathfrak{p} J\right)_{\mathfrak{p}}$ for $j=l+1, \ldots, t$. Hence there exists $\alpha \in R \backslash \mathfrak{p}$ and matrices $U_{i}$, with entries in $R$ for all $i$ and entries of $U_{2}$ in $\mathfrak{p}$, such that $\alpha \mathbf{a}=\mathbf{a}^{\prime} U$ for

$$
U=\left[\begin{array}{cccc}
\alpha I_{l} & U_{1} & 0 & U_{3} \\
0 & U_{2} & \alpha I_{m} & U_{4}
\end{array}\right] .
$$

Let $X^{\prime}=U X$. By Lemma 2.8 there is a $1 \times g$ vector $\mathbf{e}$ with entries in $R^{\prime}=$ $R \llbracket X \rrbracket_{P \cap R \llbracket X \rrbracket}$ such that $K_{P}=\left(\mathbf{b}, \mathbf{e}+v \mathbf{a}^{\prime}\right)$ and $\mathbf{e} X^{\prime}=\beta \mathbf{b}$ for some $\beta \in R^{\prime}$. If $\beta+\alpha v$ is not in $P$, then $\left(\mathbf{e}+v \mathbf{a}^{\prime}\right) X^{\prime}=(\beta+\alpha v) \mathbf{b}$ implies that $K_{P}=\left(\mathbf{e}+v \mathbf{a}^{\prime}\right)$ is a complete intersection. Henceforth we assume $\beta+\alpha v$ is in $P$.

Claim. If $l+1 \leqslant r \leqslant g$ and $P$ contains $I_{r}\left(X^{\prime}\right)$, then

$$
\text { ht } P \geqslant \text { ht } \mathfrak{p}+1+(g-r+1)(g-r) \text {. }
$$


If we partition $X$ compatibly with $U$,

$$
\left.X=\left[\begin{array}{ccc}
I_{l} & 0 & 0 \\
0 & I_{t-l} & 0 \\
Y_{1} & Y_{3} & Y_{5} \\
Y_{2} & Y_{4} & Y_{6}
\end{array}\right]\right\} \quad n \quad l-t-m
$$

then $I_{r}\left(X^{\prime}\right) \equiv \alpha^{r} I_{r-1}\left(\left[Y_{3}, Y_{5}\right]\right)$ modulo $\left(\mathfrak{p}, Y_{1}, Y_{2}, Y_{4}, Y_{6}\right)$. This ideal has height $(m-(r-l)+1)(g-1-l-(r-l)+1)=(g-r+1)(g-r)$ in $\left(R_{\alpha} / \mathfrak{p}\right)\left[Y_{3}, Y_{5}\right]$ by the same arguments as before. Since $\beta+\alpha v$ is in $P$ but not in $\left(P \cap R[X]_{\alpha}\right) S_{\alpha}$, the estimate of Claim 1 above can be improved by +1 .

If $P$ actually contains $I_{g-2}\left(X^{\prime}\right)$, then ht $P \geqslant$ ht $\mathfrak{p}+7 \geqslant g+7$, contrary to the hypothesis that ht $P \leqslant g+6$. Some $(g-2) \times(g-2)$ minor of $X^{\prime}$ therefore becomes invertible in $S_{P}$, and we can use the equation $\left(\mathbf{e}+v \mathbf{a}^{\prime}\right) X^{\prime}=(\beta+\alpha v) \mathbf{b}$ to solve for $g-2$ of the $e_{i}+v a_{i}^{\prime}$ in terms of the remaining two entries and $\mathbf{b}$. Thus $\mu\left(K_{P}\right) \leqslant g+1$, but once again by Kunz we conclude $K_{P}$ is a complete intersection.

CoRollaRy 6.5. (1) Let $A=R / J$ be a codimension $g$ Gorenstein quotient of a power series ring $R$ over $k$. Let $S=R \llbracket X, v \rrbracket$ and $B=S / K$ be the general double link of $A$ as in Definition 2.5. Assume that $A$ is a rigid domain regular in codimension $i$ for $i \leqslant 6$. Then $B$ is a rigid domain regular in codimension $i$. In particular, the algebras $A(l, \tau)$ satisfy $\left(R_{6}\right)$.

(2) Each algebra $B(\tau)$ satisfies $\left(R_{6}\right)$.

Proof. (1) Apply Corollary 5.2 and Theorems 6.4 and 6.1 to $B$, and Proposition 3.1 to $A(l, \tau)$.

(2) Since $B^{\prime}(\tau)=B(\tau) \llbracket x_{21}, \ldots, x_{\tau 1} \rrbracket$ (see Example 3.2) it suffices to show that $B^{\prime}(\tau)$ satisfies $\left(R_{6}\right)$. Now $B^{\prime}(\tau)$ is constructed from $A(0, \tau)$ by using $X$ as in Theorem 6.4 with $t=1$; since $A(0, \tau)$ satisfies $\left(R_{6}\right)$ it is a complete intersection at primes of height at most six, and so therefore is $B^{\prime}(\tau)$. By Corollary $5.3, B^{\prime}(\tau)$ is rigid. Application of Theorem 6.1 concludes the proof.

RemarK. In light of Proposition 2.7 this result includes [4, Proposition 6.1] when $g=3$. For $l=1$ it recovers, and improves, our Theorem 2.7 and following remarks in [24].

For our final application of Theorem 6.3 we recall several definitions. The symmetric algebra of an $R$-module $M$ is denoted $\operatorname{Sym}_{R}(M)$. The Rees algebra of an ideal $I$ is $\mathcal{R}(I)=R \oplus I \oplus I^{2} \oplus \cdots$ and $\operatorname{gr}_{I}(R)=\oplus I^{n} / I^{n+1}$ is the associated graded algebra. If a generates $I$, then $H_{i}(I ; R)$ denotes the homology of the Koszul complex on the given generators of $I$. If $H_{i}(I ; R)$ is a Cohen-Macaulay $R$-module for one generating set of $I$, then it is a Cohen-Macaulay module for any generating set.

CoRollary 6.6. If $R=R(l, \tau)$ and $A=A(l, \tau)=R / I$, then:

(1) The $R$-algebras $\operatorname{Sym}_{R} I$ and $R(I)$ are Cohen-Macaulay and isomorphic.

(2) The A-algebras $\operatorname{Sym}_{A}\left(I / I^{2}\right)$ and $\operatorname{gr}_{I}(R)$ are isomorphic Gorenstein normal domains. 
(3) The Koszul homology modules $H_{i}(I ; R)$ are Cohen-Macaulay for all $i \geqslant 0$.

(4) The symbolic power $I^{(k)}$ is equal to $I^{k}$ for all $k \geqslant 1$.

Proof. Inasmuch as each $A$ is a domain in the linkage class of a complete intersection and $I$ satisfies $\left(\mathrm{LG}_{4}\right)$, the first three assertions are a direct application of Huneke's version [18, Theorems 1.16, 1.18 and 1.14] of a theorem due to Herzog, Simis and Vasconcelos [14]. The last statement follows from (2).

7. Resolution invariants; classification of $A(l, \tau)$ and $B(\tau)$ into distinct Herzog classes. Let $k$ be an algebraically closed field and $P$ be a power series ring $k \llbracket X \rrbracket$ in finitely many indeterminates $X_{1}, \ldots, X_{m}$ (we do not fix $m$ ). In this section we consider only algebras of form $A=P / I$ and we denote the collection of all such by Q. Following Herzog [13], we write $B \sim A$ if there is an algebra $C$ in $Q$ which contains regular sequences $\mathbf{x}$ and $\mathbf{y}$ such that $A \cong C /(\mathbf{x})$ and $B \cong C /(\mathbf{y})$. This relation is preserved under power series extension $\left(B \llbracket Y_{1}, \ldots, Y_{s} \rrbracket \sim B\right)$, specialization by a regular sequence $(B \sim B /(\mathbf{z}))$; and if $\theta: k \llbracket \varepsilon_{1}, \ldots, \varepsilon_{s} \rrbracket \rightarrow B^{\prime}$ is a formal deformation of $B$, then by Proposition $B$ we conclude that $B^{\prime} \sim B$ since $B \cong$ $B^{\prime} /$ (regular sequence).

Herzog [13] showed that $\sim$ is an equivalence relation on a certain subclass $\delta$

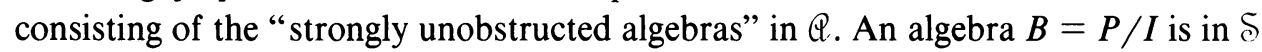
if $B$ is Cohen-Macaulay, reduced, and $I \otimes_{P} K_{B}$ is a Cohen-Macaulay $B$-module, where $K_{B}$ is the canonical module of $B$. We write $B \in[A]$ if $A$ and $B$ are in $\delta$ and $B \sim A$. We call $[A]$ a Herzog class.

Proposition 7.1. If $A=P / I$ is reduced and in the linkage class of a complete intersection, then $A$ is in $\mathcal{S}$. In particular, each algebra $A(l, \tau)$ and $B(\tau)$ is in $\mathcal{S}$.

Proof. By hypothesis, $A$ is Cohen-Macaulay. If $\mathfrak{p}$ is a minimal prime of $A$, then since $A$ is reduced, $A_{\mathrm{p}}$ is regular and hence a complete intersection. In other words $A$ is a generic complete intersection. Buchweitz [5, 6.2.11] has shown that under these conditions $\operatorname{Tor}_{1}^{P}\left(A, K_{A}\right)=I \otimes_{P} K_{A}$ is a Cohen-Macaulay $A$-module.

If $B$ is in $\mathcal{S}$, then $T^{2}(B / k, B)=0$ (see Herzog [13, Satz 1.4]). In particular, any first order deformation of $B$ is unobstructed and may be extended to give an analytic deformation $k \llbracket T_{1}, \ldots, T_{s} \rrbracket \rightarrow B^{\prime}$ of $B$ with $B^{\prime}$ in $\mathcal{S}$. If $\xi_{1}, \ldots, \xi_{s}$ generate $T^{\mathrm{l}}(B / k, B)$ as a $B$-module and $B^{\prime}$ is obtained from $B$ by deforming in the directions $\xi_{1}, \ldots, \xi_{s}$, then $B^{\prime}$ is a rigid $k$-algebra in [B]. (See the proof of Herzog [13, Satz 2.3(b)] and recall that $B^{\prime} \sim B$ since $B \cong B^{\prime} /$ (regular sequence).) The following lemma enables us to show that there is a distinguished representative of a class $[B]$, the so-called "generic" algebra for $[B]$. Recall that the embedding dimension of $A$ is defined to be $\mu\left(\mathrm{m}_{A}\right)=\operatorname{dim}_{k}\left(\mathrm{~m}_{A} / \mathrm{m}_{A}^{2}\right)$ and the embedding codimension, emb.cod. $A$, is defined to be $\mu\left(\mathrm{m}_{A}\right)-\operatorname{dim} A$. If $\mu\left(\mathrm{m}_{A}\right)=m$, then $A$ can be written as $P / I$ with $\operatorname{dim} P=m$ and ht $I=$ emb.cod. $A$.

Lemma 7.2. Let $A$ and $B$ be rigid algebras in S. If $B \in[A]$ and $s=\operatorname{dim} B-\operatorname{dim} A$ $\geqslant 0$, then $B \cong A \llbracket Y_{1}, \ldots, Y_{s} \rrbracket$.

Proof. See the proof of 2.3(d) in Herzog [13]. 
Definition 7.3. If $A$ is a rigid algebra in $\delta$ of smallest embedding dimension among all the rigid algebras in [ $A$ ], then $A$ is called generic for the class [ $A]$.

The reason for the terminology is that any other algebra in $[A]$ is a "specialization" of $A$, in a sense made precise in the following proposition. The last two points are needed because it is the derivation property which we prove is preserved under suitable linkage, rather than the generic property per se.

Proposition 7.4. Let $A$ and $B$ be rigid algebras in $[A]$.

(1) If $A$ is generic, then $B=A \llbracket Y_{1}, \ldots, Y_{s} \rrbracket$.

(2) If $A$ is generic and $C$ is any other algebra in $[A]$, then $C \cong A \llbracket Y_{1}, \ldots, Y_{t} \rrbracket /$ (regular sequence).

(3) If the image of every $k$-derivation $d: A \rightarrow A$ is contained in $\mathrm{m}_{A}$, then $A$ is generic.

(4) If char $k=0$ and $A$ is generic, then the image of every $k$-derivation $d: A \rightarrow A$ is contained in $\mathrm{m}_{A}$.

Proof. Statement (1) follows from the definition and Lemma 7.2.

(2) By the discussion following Proposition 7.1 there is a rigid algebra $C^{\prime}$ that specializes to $C$ by a regular sequence. Apply (1) to $C^{\prime}$.

(3) If $A$ is not generic, then there is a rigid algebra $C$ in $[A]$ with smaller embedding dimension. By the lemma $A \cong C \llbracket Y_{1}, \ldots, Y_{s} \rrbracket$. The image of the derivation $\partial / \partial Y_{1}$ is not contained in $\mathrm{m}_{A}$.

(4) If $d: A \rightarrow A$ is a $k$-derivation with $d(z)$ a unit for some $z \in A$, then by Lipman [25, Theorem 2], there is a subring $A^{\prime} \subset A$ such that $A=A^{\prime} \llbracket z \rrbracket$. Then $A^{\prime}$ is a rigid algebra in $[A]$ with smaller embedding dimension than $A$. Consequently, $A$ is not generic.

RemarK 7.5. If $A=P / I$ then there is a natural inclusion $\operatorname{Der}_{k}(A, A) \hookrightarrow$ $\operatorname{Der}_{k}(P, A)$. Since $A$ is a finitely generated $P$-module we have:

(1) Given $a_{1}, \ldots, a_{m}$ in $A$, there is a $k$-derivation $d: P \rightarrow A$ with $d(p)=\sum \partial p a_{i} / \partial X_{i}$.

(2) If $d: P \rightarrow A$ is a $k$-derivation with $d\left(X_{i}\right)=a_{i}$, then $d(p)=\Sigma \partial p a_{i} / \partial X_{i}$.

(3) A derivation $d \in \operatorname{Der}_{k}(A, A)$ is in $\operatorname{m}_{A} \operatorname{Der}_{k}(P, A)$ if and only if the image of $d$ is contained in $\mathrm{m}_{A}$.

(4) Any derivation $d \in \operatorname{Der}_{k}(A, A)$ can be viewed as a derivation $D: P \rightarrow P$ such that $D(I) \subset I$.

From our point of view, however, the generic algebra of a class may not in and of itself be the appropriate object to study. We are interested in classifying algebras of fixed codimension $g$; i.e. algebras of form $P / I$ with ht $I=g$ (and especially Gorenstein algebras with $g=4$ ). The generic algebra for all complete intersections, for instance, is simply $k$, which lacks the structure (e.g. finite free resolution together with associated invariants) that a particular presentation $P / I=k$ carries.

Definition 7.6. Suppose $A$ is in $\mathcal{Q}$ and emb.cod. $A \leqslant g$. Write $A=P / I$ with ht $I=g$. Let $\Lambda_{\bullet}$ be the graded $k$-algebra $\operatorname{Tor}_{\bullet}^{P}(A, k)$. Then define

$$
\begin{gathered}
\delta_{1}(A)=\operatorname{dim}_{k} \Lambda_{1} \Lambda_{1}, \quad \delta_{2}(A)=\operatorname{dim}_{k} \Lambda_{1} \Lambda_{2}, \\
\delta_{0}(A)=\mu(I)=\operatorname{dim}_{k}\left(I / \mathrm{m}_{P} I\right), \quad \nu(A)=\mu\left(\left(I / I^{2}\right)^{*}\right) .
\end{gathered}
$$


Here $\mu(M)$ is the minimal number of generators of the $P$-module $M$, and $-^{*}=$ $\operatorname{Hom}_{A}(-, A)$. None of these definitions depends on the particular representation $P / I$ as long as ht $I=g$.

The following lemma enables us to compute these invariants for all algebras in a given class if we can compute them for a particular algebra in that class.

Lemma 7.7. Let $B$ be an algebra in $\delta$ with emb.cod. $B \leqslant g$. Then $[B]$ has a generic algebra $A$ with emb. cod. $A=a \leqslant g$. There are power series rings $P=k \llbracket X_{1}, \ldots, X_{m} \rrbracket$ and $R=k \llbracket T_{1}, \ldots, T_{q} \rrbracket$ containing ideals $I$ and $J$, respectively, such that $A \cong P / I$, $B \cong R / J$, and ht $I=$ ht $J=g$. Moreover, there is a local $k$-algebra map $P \rightarrow R$ such that $B \cong R \otimes_{P} A$.

Proof. By the discussion following Propositions 7.1 and 7.4 we know there is a generic algebra $A$ and $B \cong A \llbracket Y_{1}, \ldots, Y_{t} \rrbracket /(\mathbf{z})$ where $Y_{1}, \ldots, Y_{t}$ are indeterminates and $\mathbf{z}=z_{1}, \ldots, z_{p}$ is a regular sequence. Then

$$
a=\text { emb.cod. } A=\text { emb. cod. } A \llbracket Y_{1}, \ldots, Y_{t} \rrbracket \leqslant \text { emb. cod. } B \leqslant g
$$

and $A=P^{\prime} / I^{\prime}$, where $P^{\prime}=k \llbracket X_{1}, \ldots, X_{n} \rrbracket$, ht $I^{\prime}=a$, and $I^{\prime} \subset(X)^{2}$. Thus we can write $A=P / I$ with $P=k \llbracket X_{1}, \ldots, X_{m} \rrbracket, I=\left(I^{\prime}, X_{n+1}, \ldots, X_{m}\right)$, and $m-n=g-a$. Set $R^{\prime}=P^{\prime} \llbracket Y_{1}, \ldots, Y_{t} \rrbracket$. Let us suppose (after possibly reindexing) that $z_{1}, \ldots, z_{r}$ are in $\left(\mathrm{m}_{A}, Y_{1}, \ldots, Y_{t}\right)^{2}$ and that $z_{r+1}, \ldots, z_{p}$ begin a minimal generating set for $\left(\mathrm{m}_{A}, Y_{1}, \ldots, Y_{t}\right)$. Lift all $z_{i}$ to $Z_{i}$ in $R^{\prime}$. Then $\mathrm{m}_{R^{\prime}}$ has a minimal set of generators $\left\{Z_{r+1}, \ldots, Z_{p}, T_{1}, \ldots, T_{l}\right\}$ where $n+t=p-r+l$. Observe that $B=$ $R^{\prime} /\left(I^{\prime}, Z_{1}, \ldots, Z_{p}\right)$ can be re-expressed as $k \llbracket T_{1}, \ldots, T_{l} \rrbracket / J^{\prime}$, and this is a minimal presentation since $\mu\left(\mathrm{m}_{B}\right)=l$. It follows that ht $J^{\prime}=$ emb. $\operatorname{cod} . B=r+a$. If $R=$ $k \llbracket T_{1}, \ldots, T_{q} \rrbracket$ with $q=g-r-a+l$, then $B=R /\left(J^{\prime}, T_{l+1}, \ldots, T_{q}\right)$ is the desired presentation $B=R / J$. It is straightforward to define a map of $k$-algebras such that $J=I R$ and $B=R \otimes_{P} A$.

Conversely, it is easy to see that if $A=P / I$ is Cohen-Macaulay, $B=R / J$ with ht $I=$ ht $J$, and there is a map $P \rightarrow R$ such that $B \simeq R \otimes_{P} A$, then $B \sim A$.

THEOREM 7.8. If $B$ is $\varsigma$ and emb.cod. $B \leqslant g$, then $\delta_{1}(B)=\delta_{1}(A), \delta_{2}(B)=\delta_{2}(A)$, $\delta_{0}(B)=\delta_{0}(A)$, and $\nu(B)=\nu(A)$, where $A$ is generic for $[B]$.

Proof. By the lemma we may assume that $A=P / I$ and $B=R / J=R \otimes_{P} A$ with ht $J=$ ht $I=g$. Let $\mathbf{F}$ be a minimal free resolution of $A$ over $P$. Since $A$ is perfect, the change of rings is local, and ht $J=$ ht $I$, Hochster's theorem on generic specialization [16, Proposition 6.14] implies that $R \otimes_{P} \mathbf{F}$ is a minimal free resolution of $B$ over $R$. Hence

$$
\operatorname{Tor}_{\mathbf{C}}^{P}(A, k)=\mathbf{F} \otimes_{P} k=\left(\mathbf{F} \otimes_{P} R\right) \otimes_{R} k=\operatorname{Tor}_{\bullet}^{R}(B, k) .
$$

Consequently, $\delta_{1}(A)=\delta_{1}(B), \delta_{2}(A)=\delta_{2}(B)$, and $\delta_{0}(A)=\delta_{0}(B)$.

To prove the last assertion we note that $B$ is reduced, hence satisfies $R_{0}$, and hence is a generic complete intersection. It follows that $J / J^{2}$ is free of rank $g$ at the minimal primes of $B$, and so $\operatorname{rank}\left(J / J^{2}\right)^{*}=g$. The same observations hold for $A$ and $I$; the result follows immediately then form Herzog [13, Satz 2.3e].

The $\nu$-invariant has a particularly simple and attractive interpretation. 
Proposition 7.9. Let $A=P / I$ be generic and suppose ht $I=g$. If char $k \neq 0$, assume that the image of every $k$-derivation $A \rightarrow A$ is contained in $\mathrm{m}_{A}$. Then $\nu(A)=\operatorname{dim} P$. In particular $\nu(A)=\varepsilon_{0}(A)$, the embedding dimension of $A$, if emb. cod. $A=g$.

REMARK. If char $k=0$ the derivation hypothesis is automatic by Proposition 7.4.

Proof. Applying $\operatorname{Hom}_{A}(-, A)$ to the exact sequence

$$
I / I^{2} \rightarrow \Omega_{P / k} \otimes_{P} A \rightarrow \Omega_{A / k} \rightarrow 0,
$$

we obtain an exact sequence

$$
0 \rightarrow \operatorname{Der}_{k}(A, A) \rightarrow \operatorname{Der}_{k}(P, A) \rightarrow\left(I / I^{2}\right)^{*} \rightarrow T^{1}(A / k, A) \rightarrow 0 .
$$

Since $A$ is rigid, $T^{1}(A / k, A)=0$, and we are done by Remark 7.5 and Nakayama's lemma.

The invariant $\delta_{1}$ is more subtle. Let $\mathbf{F}$ be a minimal free resolution of $A$ over $P$ with differential $d$, and let $e_{1}, \ldots, e_{n}$ be a basis for $F_{1}$. An element $\left(d e_{i}\right) e_{j}-\left(d e_{j}\right) e_{i}$ in $F_{1}$ (with $i \neq j$ ) is called a Koszul relation. Each of these is in $\operatorname{Im}\left(d_{2}\right)$. For a proof of the following result see [24].

Proposition 7.10. If $A=P / I$ with ht $I=g$, then $\delta_{1}(A)$ is the maximum number of Koszul relations that can be used in a minimal generating set for $\operatorname{Im}\left(d_{2}\right)$, i.e. that can be taken as columns in a matrix representation of $d_{2}$.

Since we will not actually need to use $\delta_{2}$ to distinguish the algebras discussed in this section we omit the details of its computation. As it is sufficient to compute the four resolution invariants for the generic algebra of a class, we must be able to identify the generic algebra. The following result shows that the property of being generic is preserved under general double link.

THEOREM 7.11. Let $R=k \llbracket Z_{1}, \ldots, Z_{p} \rrbracket, A$ be a codimension $g$ Gorenstein quotient of $R$, and $B$ be the special double link of $A$. Assume that $A$ is generic and that $\operatorname{Im}(\beta) \subset \mathrm{m}_{A}$ for every $k$-derivation $\beta: A \rightarrow A$. Then $B$ is generic and $\operatorname{Im}(\beta) \subset \mathrm{m}_{B}$ for every $k$-derivation $\beta: B \rightarrow B$.

Proof. By definition there is a $1 \times n$ matrix a with entries in $R$, an $n \times(g-1)$ matrix of indeterminates $X$, and an indeterminate $v$ such that $A=R /(a)$ and $B=P / K$, where $P=R \llbracket X, v \rrbracket$ and $K=(\mathbf{a} X, \mathbf{c}+v \mathbf{a})$. If $P$ is graded as in Proposition 2.6 , then each entry of $\mathbf{a} X$ has degree 1 and each entry of $\mathbf{c}+v \mathbf{a}$ has degree $g-1$. If $f \in P$, then we write $f=\sum f_{(i)}$, where $f_{(i)}$ is a form of degree $i$.

By Corollary 5.2, $B$ is rigid. By Proposition 7.4 and Remark 7.5 it suffices to show that if $\beta: P \rightarrow P$ is a $k$-derivation with $\beta(K) \subset K$, then $\operatorname{Im}(\beta) \subset \mathrm{m}_{P}$. Since $g \geqslant 3$, it is clear that $f_{(0)}=0$ and $f_{(1)} \in(\mathbf{a} X)$ if $f \in K$.

Let $\left(\beta a_{i}\right)_{(0)}=r_{i},\left(\beta x_{i j}\right)_{(0)}=r_{i j}$, and $\left(\beta x_{i j}\right)_{(1)}$ be the linear form $p_{i j}$ in $R \llbracket X \rrbracket$. Since $\beta \sum a_{i} x_{i j}=\sum\left(\beta a_{i}\right) x_{i j}+\sum a_{i}\left(\beta x_{i j}\right)$ is in $K$, we have $\left(\beta \sum a_{i} x_{i j}\right)_{(1)}=\sum r_{i} x_{i j}+$ $\sum a_{i} p_{i j}$ in (a $\left.X\right)$. It follows that each $r_{i}$ is in (a).

Define $\beta^{\prime}: R \hookrightarrow P \stackrel{\beta}{\rightarrow} P \rightarrow R$ using the natural inclusion and projection. This is a derivation and $\beta^{\prime}\left(a_{i}\right)=r_{i}$, so $\beta^{\prime}(\mathbf{a}) \subset$ (a) and hence $\beta^{\prime}$ induces a $k$-derivation $A \rightarrow A$. By hypothesis, $\operatorname{Im}\left(\beta^{\prime}\right) \subset \mathfrak{m}_{R}$. Hence $\operatorname{Im}\left(\left.\beta\right|_{R}\right) \subset \mathfrak{m}_{P}$. 
To show $\operatorname{Im}(\beta) \subset \mathrm{m}_{P}$, we will show that $\beta x_{i j}$ and $\beta v$ are in $\mathfrak{m}_{P}$. Let

$$
\cdots \rightarrow P^{m} \stackrel{d_{2}}{\rightarrow} P^{n} \stackrel{\mathrm{a}}{\rightarrow} P
$$

be the start of a minimal free resolution of $A$. Since $0=\left(\beta \sum a_{i} x_{i j}\right)_{(0)}=\sum a_{i} r_{i j}$ it follows that $\left[r_{1 j}, \ldots, r_{n j}\right]^{t}$ is in the column space of $d_{2}$ for each index $j$. Thus all $r_{i j}$ are in $\mathfrak{m}_{R}$ and $\beta\left(x_{i j}\right)$ are in $\mathfrak{m}_{P}$. Finally,

$$
\beta\left(c_{i}+v a_{i}\right)=\beta c_{i}+v \beta a_{i}+(\beta v) a_{i}
$$

is in $K \subset(X, v)$. Since $c_{i}$ is an $R$-linear combination of maximal order minors of $X$ and $g-1 \geqslant 2$, we have $\beta c_{i} \in(X)$ and $(\beta v) a_{i} \in(X, v)$. It follows that $(\beta v)_{0} a_{i}=0$ for all $i$ and consequently $\beta v \in(X, v) \subset \mathrm{m}_{P}$.

We turn now to the classification of codimension four Gorenstein algebras. For the remainder of this section $g=4$.

Corollary 7.12. Each algebra $A=A(l, \tau)$ is generic. If $\beta: A \rightarrow A$ is a $k$-derivation, then $\operatorname{Im}(\beta) \subset \mathrm{m}_{A}$.

Proof. Apply Proposition 3.1(3), and Theorem 7.11. The statement is clearly true for $k$.

Once again, because the $B(\tau)$ are not general double links, we take a direct computational approach.

LEMMA 7.13. Let $R$ be a power series ring over $k$ and a be $a 1 \times n$ vector which minimally generates a grade $g \geqslant 4$ Gorenstein ideal in $R$. For any $r, 0 \leqslant r \leqslant n-1$, let $X$ and $Z$ be $r \times 1$ and $(n-1) \times(g-2)$ matrices with indeterminate entries, $P=$ $R \llbracket X, Z, v \rrbracket$, and $\mathbf{b}=\mathbf{a} M$ for

$$
M=\left[\begin{array}{l|l}
1 & 0 \\
\hline 0 & \\
\hline X & Z
\end{array}\right] .
$$

Assume that $\mathbf{b}$ is a regular sequence and that $\mathbf{c}$ in (2.2) is obtained by a map of associative $D G$ algebras. Let $K=(\mathbf{b}, \mathbf{c}+v \mathbf{a})$. Suppose that $\operatorname{Im}\left(\beta^{\prime}\right) \subset \mathrm{m}_{R}$ for every $k$-derivation $\beta^{\prime}: R \rightarrow R$ such that $\beta^{\prime}(\mathbf{a}) \subset(\mathbf{a})$. Then $\beta(R \llbracket Z, v \rrbracket) \subset \mathrm{m}_{P}$ for every $k$-derivation $\beta: P \rightarrow P$ such that $\beta(K) \subset K$.

Proof. Let $S=R \llbracket X \rrbracket$. Assign degrees in $P$ as follows: $\operatorname{deg} S=0, \operatorname{deg} z_{i j}=1$, $\operatorname{deg} v=g-2$. Then $\operatorname{deg} b_{1}=0, \operatorname{deg} b_{j}=1$ for $2 \leqslant j \leqslant g-1$, and $\operatorname{deg}\left(c_{i}+v a_{i}\right)=$ $g-2$ for $1 \leqslant i \leqslant n$. If $f$ is in $K$, then $f_{(0)} \in\left(b_{1}\right) S$ and $f_{(1)} \in\left(b_{1}\left\{z_{i j}\right\}, b_{2}, \ldots, b_{g-1}\right) S$. Let $\beta^{\prime}$ be the composition derivation $R \hookrightarrow P \stackrel{\beta}{\rightarrow} P \rightarrow R$. The proof is completed by showing that $\beta^{\prime}(\mathbf{a}) \subset$ (a) and consequently $\beta(R) \subset \mathfrak{p}_{P}, \beta(v) \in \mathfrak{m}_{P}$, and $\beta\left(z_{i j}\right) \in$ $\mathfrak{m}_{P}$. One uses degree arguments exactly as in the proof of Theorem 7.11 above.

Corollary 7.14. Each algebra $B=B(\tau)$ with $\tau \geqslant 5$ is generic. If $\beta: B \rightarrow B$ is $a$ $k$-derivation, then $\operatorname{Im}(\beta) \subset \mathfrak{m}_{B}$.

Proof. By Corollary 5.3 we know that $B$ is rigid. By Proposition 7.4 it suffices to prove the derivation property. Recall that $B(\tau)$ has the form $P / K$ in the lemma, 
where $n=\tau+1, r=1$, and $a_{i}=Y_{i}$ are maximal pfaffians of a $\tau \times \tau$ alternating matrix $Y$ for $1 \leqslant i \leqslant \tau$. It only remains to verify that $\beta\left(x_{\tau+1}\right) \in \mathrm{m}_{P}$. Since $b_{1}=Y_{1}$ $+a_{\tau+1} x_{\tau+1}$ is in $K,\left(\beta b_{1}\right)_{(0)}=b_{1} s$ for some $s \in S$; hence

$$
b_{1} s=\left(\beta Y_{1}\right)_{(0)}+a_{\tau+1}\left(\beta x_{\tau+1}\right)_{(0)}=\left(\beta a_{\tau+1}\right)_{(0)} x_{\tau+1} .
$$

Since $\tau \geqslant 5$ each $Y_{i}$ is at least quadratic in the $y_{i j}$, so $\beta Y_{1}$ is in $\left(y_{i j}\right) S$. Then

$$
0 \equiv a_{\tau+1}\left(\beta x_{\tau+1}\right)_{(0)} \text { modulo }\left(y_{i j}, x_{\tau+1}\right) \text {. }
$$

In the construction of $B(\tau)$ the element $a_{\tau+1}$ is independent of the other indeterminates and, in particular, not a zero divisor on $\left(y_{i j}, x_{\tau+1}\right)$. Consequently, $\left(\beta x_{\tau+1}\right)_{(0)}$ is in $m_{S}$ and hence $\beta x_{\tau+1}$ is in $m_{P}$.

THEOREM 7.15. If $A \in[A(l, \tau)]$ with $l \geqslant 0$, and $\tau \geqslant 3$ odd, then

$$
\begin{gathered}
\delta_{1}(A)= \begin{cases}6 & \text { if } l=0 \text { and } \tau=3, \\
\tau & \text { if } l=0 \text { and } \tau \geqslant 5, \\
3 & \text { if } l \geqslant 1 ;\end{cases} \\
\delta_{0}(A)=3 l+\tau+1 ; \\
\nu(A)=\nu(A(l-1, \tau))+3 \delta_{0}(A(l-1, \tau))+1 \\
=\tau(\tau-1) / 2+3 l(\tau+1)+l+1+9 l(l-1) / 2 .
\end{gathered}
$$

If $B \in[B(\tau)]$ with $\tau \geqslant 5$ odd, then $\delta_{1}(B)=3, \delta_{0}(B)=\tau+3$, and $\nu(B)=$ $\tau(\tau-1) / 2+3+2 \tau$. Consequently, the Herzog classes of these algebras $A(l, \tau)$ and $B(\tau)$ are distinct. (Note $B(3) \in[A(0,5)]$ by Example 3.2.)

REMARK. The algebras $A(2,3), B(7)$ and $A(0,9)$ all have resolutions

$$
0 \rightarrow P \rightarrow P^{10} \rightarrow P^{18} \rightarrow P^{10} \rightarrow P
$$

(a different $P$ in each case). Yet none of the resolutions can be obtained from the others under a change of rings homomorphism.

Proof. By Theorem 7.8 each of these invariants takes the same value for all algebras in a given Herzog class, so we may as well assume $A=A(l, \tau)$ and $B=B(\tau)$. The invariants $\delta_{0}(A)$ and $\delta_{0}(B)$ are calculated in Corollary 3.6. Since $A(l, \tau)=R(l, \tau) / I$ with ht $I=4$, we may apply Proposition 7.9 and Corollary 7.12 to see that $\nu(A(l, \tau))=\operatorname{dim} R(l, \tau)$. If $l=0$, then $R(0, \tau)=k \llbracket Y, w \rrbracket$, where $Y$ consists of $\tau(\tau-1) / 2$ indeterminates; hence $\nu(A(0, \tau))=\tau(\tau-1) / 2+1$. Assume inductively that (4) holds for $A(l, \tau)$. Then $R(l+1, \tau)=R(l, \tau) \llbracket X, v \rrbracket$, where $X$ consists of $3 \delta_{0}(A(l, \tau))$ indeterminates. Hence

$$
\nu(A(l+1, \tau))=\operatorname{dim} R(l+1, \tau)=\nu(A(l, \tau))+3 \delta_{0}(A(l, \tau))+1
$$

and the explicit formula is readily verified using (3). We compute $\nu(B(\tau))$ similarly.

Next we calculate $\delta_{1}(A)$. If $A=A(0,3)$, then $A$ is resolved by a free exterior algebra $\wedge 9\left(R^{4}\right)$, so $\delta_{1}(A)=6$. If $A=A(0, \tau)$ for $\tau \geqslant 5$, then the minimal free resolution of $A$ begins

$$
P^{2 \tau} \stackrel{d_{2}}{\rightarrow} P^{\tau+1} \stackrel{d_{1}}{\rightarrow} P
$$


with $d_{1}=[\mathbf{y}, w]=\left[Y_{1}, \ldots, Y_{\tau}, w\right]$ and

$$
d_{2}=\left[\begin{array}{cc}
Y & w I \\
0 & -\mathbf{y}
\end{array}\right]
$$

where $Y$ is a $\tau \times \tau$ alternating matrix of indeterminates and $Y_{i}=\operatorname{Pf}_{i}(Y)$. Clearly, the last $\tau$ columns of $d_{2}$ represent Koszul relations, so $\delta_{1}(A) \geqslant \tau$ by Proposition 7.10, and $\left\{e_{i} e_{\tau+1} \mid 1 \leqslant i \leqslant \tau\right\}$ is part of a basis for $F_{2}$. Each of the other products $e_{i} e_{j}$ is in $\mathrm{m} F_{2}$ for $1 \leqslant i, j \leqslant \tau$ because $Y\left[Y_{1 i j}, \ldots, Y_{\tau i j}\right]^{t}=Y_{j} e_{i}-Y_{i} e_{j}$ (see [24, Lemma 1.2]). Since $\tau \geqslant 5$, each lower order pfaffian $Y_{l i j}$ is in $\mathrm{m}_{R}$. It follows that $\delta_{1}(A)=\tau$.

The calculations prior to Corollary 3.6 show that if $A=A(l, \tau)$ with $l \geqslant 1$, then the minimal presentation of $A$ is

$$
P^{2 n+4} \stackrel{d_{2}}{\rightarrow} P^{n} \stackrel{d_{1}}{\rightarrow} P
$$

with $P=R(l, \tau), d_{1}=[\mathbf{b}, \mathbf{c}+v \mathbf{a}]$ and

$$
d_{2}=\left[\begin{array}{ccc}
v I & B & M^{t} s^{-1} \\
-X & 0 & -A
\end{array}\right]
$$

where $R^{2 n-2} \stackrel{A}{\rightarrow} R^{n} \stackrel{\text { a }}{\rightarrow} R$ is a minimal presentation of $A(l-1, \tau)$ with $R=R(l-1, \tau)$. As in Proposition 2.6 we give $P=R \llbracket X, v \rrbracket$ a grading by assigning $\operatorname{deg} R=0$, $\operatorname{deg} x_{i j}=1$, and $\operatorname{deg} v=3$. Then the degree of each entry of $M^{t} s^{-1}$ is 2 and the degree of each entry of $\mathbf{c}$ is 3 . The vector $\mathbf{b}$ is equal to $\mathbf{a} X$ and each entry has degree 1. The columns of

$$
B=\left[\begin{array}{ccc}
0 & b_{3} & -b_{2} \\
-b_{3} & 0 & b_{1} \\
b_{2} & -b_{1} & 0
\end{array}\right]
$$

represent Koszul relations on $d_{1}$, so $\delta_{1}(A) \geqslant 3$.

Suppose $\gamma$ is in $P^{2 n+4}$, and $d_{2} \gamma$ is a linear combination Koszul relations including at least one that involves an entry from $\mathrm{c}+v \mathbf{a}$. Write

$$
\gamma=\left[\begin{array}{l}
\lambda_{0}+\lambda_{1} \\
\mu_{0}+\mu_{1} \\
\eta_{0}+\eta_{1}
\end{array}\right]
$$

with $\lambda_{0}, \mu_{0}$ in $R^{3}, \lambda_{1}, \mu_{1}$ in $(X, v) P^{3}, \eta_{0}$ in $R^{2 n-2}$ and $\eta_{1}$ in $(X, v) P^{2 n-2}$. By degree considerations we have $B \mu_{0}=0, A \eta_{0}=0$, and each entry of $v \lambda_{0}$ is in $v(\mathbf{a})$. It follows that $\gamma$ is in $\mathrm{m}_{P} P^{2 n+4}$ and hence $\delta_{1}(A)=3$.

We are not able to grade $R=R(\tau)$ in order to calculate $\delta_{1}(B)$ for $B=B(\tau)$ with $\tau \geqslant 5$; thus we are forced to actually calculate the $e_{i} e_{j}$. Fortunately the multiplication table for a minimal free resolution $\mathbf{F}$ of $A(0, \tau)$ has been recorded in [24, Theorem 4.1]. In order to get a minimal presentation $G_{2} \rightarrow G_{1} \rightarrow R$ of $B$ from $\mathbf{F}$ we split off $e_{4}$ from $F_{1}$ as well as a rank 2 summand of $F_{2}$. In Example 4.2 of [24] it is observed that $e_{1} e_{2}, e_{1} e_{3}, e_{1} e_{\tau+4}$ is the start of a basis for $G_{2}$. All other products $e_{i} e_{j}$ are in $\mathrm{m} G_{2}$ because the lower order pfaffians $Y_{i j k}$ are nonunits since $\tau \geqslant 5$. Thus $\delta_{1}(B)=3$. 
To finish the proof, suppose that $A(l, \tau) \simeq A\left(l^{\prime}, \tau^{\prime}\right)$. Since $\delta_{0}(A(l, \tau))=$ $\delta_{0}\left(A\left(l^{\prime}, \tau^{\prime}\right)\right)$, we see that $\tau^{\prime}=\tau+3\left(l-l^{\prime}\right)$. On the other hand,

$$
\nu(A(l, \tau))-\nu\left(A\left(l^{\prime}, \tau+3\left(l-l^{\prime}\right)\right)\right)=l-l^{\prime} .
$$

Thus $l=l^{\prime}$ and $\tau=\tau^{\prime}$. The $B(\tau)$ with $\tau \geqslant 5$ can be distinguished from one another by $\delta_{0}$ and from the $A\left(l^{\prime}, \tau^{\prime}\right)$ by $\delta_{0}, \delta_{1}$, and $\nu$ jointly.

REMARK. One can also use $\delta_{2}$ to distinguish $B=B(\tau)$ with $\tau \geqslant 5$ from $A=A\left(l, \tau^{\prime}\right)$ with $l \geqslant 1$ and $\tau^{\prime} \geqslant 3$ since $\delta_{2}(B)=4$ whereas $\delta_{2}(A)=3$.

8. Deformation and linkage by Gorenstein ideals: observations and questions. In $§ 1$ we defined linkage by Gorenstein ideals and showed that it has the same elementary properties as ordinary linkage. In Theorem 2.1 we showed that the big from small construction of [23] can be interpreted as double linkage by Gorenstein ideals. Nevertheless, in the remaining sections we have concentrated exclusively on ordinary linkage. In this section we record differences between Gorenstein and ordinary linkage - they are dramatic and reveal why we have concentrated on ordinary linkage.

One of the main facts connecting linkage and deformaton theory is Buchweitz's result (the linchpin of our Proposition 7.1). If $A=k \llbracket X_{1}, \ldots, X_{m} \rrbracket / I$ is a reduced algebra which is in the linkage class of a complete intersection, then $T^{2}(A / k, A)=0$, i.e. $A$ is unobstructed. This result fails if we replace "linkage" by "linkage by Gorenstein". Let $A=k \llbracket T^{6}, T^{7}, T^{8}, T^{9}, T^{10} \rrbracket$ and write $A=R / I$, where $R=$ $k \llbracket X, Y, Z, W, U \rrbracket \rightarrow A$ is the coordinate map sending $X$ to $T^{6}$, etc. Let $\mathbf{a}=[X,-Y$, $Z, U]$ and $\mathbf{b}=\left[Y Z-W X, Z^{2}-W Y, Y^{2}-Z X, U Z-X^{3}, W U-X^{2} Y\right]$. Observe that $\mathbf{b}$ consists of the maximal order pfaffians of

$$
\left[\begin{array}{ccccc}
0 & -U & -X^{2} & Z & -Y \\
U & 0 & 0 & -Y & X \\
X^{2} & 0 & 0 & -W & Z \\
-Z & Y & W & 0 & 0 \\
Y & -X & -Z & 0 & 0
\end{array}\right]
$$

and that (b) is a grade 3 Gorenstein ideal contained in (a) that is not generated by a regular sequence. If we apply the construction of Theorem 2.1 to (b) $\subset$ (a) and set $v=U$, then $I=(\mathbf{b}, \mathbf{c}+v \mathbf{a})$. Using the formulas given by Buchweitz [6], it is not difficult to calculate $\operatorname{dim}_{k} T^{1}(A / k, A)=15, \operatorname{dim} T^{2}(A / k, A)=5$, and to give explicit bases. We find that the formal moduli space of $A$ is $\operatorname{Spec}\left(k \llbracket \varepsilon_{1}, \ldots, \varepsilon_{15} \rrbracket / \mathfrak{p}\right)$, where $\mathfrak{p}$ is generated by the $4 \times 4$ pfaffians of an alternating $5 \times 5$ matix in ten of the $\varepsilon_{i}$ 's. In contrast, if $B$ is a reduced $k$-algebra in the linkage class of a complete intersection, then the formal moduli space of $B$ is smooth.

There are at least two other situations in which deformation properties of linkage by Gorenstein ideals may differ from the corresponding properties of ordinary linkage. Lemma 4.1 shows that infinitesimal deformation passes over linkage by a regular sequence $(\mathbf{z})$. We do not know, and would like to know, if this result remains true when $(\mathbf{z})$ is allowed to be a Gorenstein ideal. In the same vein, Theorem 4.2 shows that rigidity of $R / I$ is preserved under linkage by the "most general" regular sequence $(\mathbf{z})$ contained in $I$. It is not clear what the "most general" $\sigma$-generated 
Gorenstein ideal in $I$ should be. Even if this issue can be resolved, there is reason to suspect that rigidity is not preserved under linkage by Gorenstein ideals.

ACKNOWLEDGEMENT. We would like to thank the referee of [23] for his questions and comments, C. Huneke for showing us his preprint [18], L. Avramov for sending us a translation of Golod's paper, and R. O. Buchweitz for his comments made in conversation with the first author while he was visiting Northeastern University. We also would like to thank the referee of the present paper for urging us to reconsider the necessity of requiring associative DG structures, as we had done in the original version. Having dropped this restrictive hypothesis, we have changed the terminology in Definition 2.5 from "special" to "general" double link.

\section{REFERENCES}

1. M. Artin, Deformations of singularities, Tata Institute for Fundamental Research, Bombay, 1976.

2. L. Avramov, Obstructions to the existence of multiplicative structures on minimal free resolutions, Amer. J. Math. 103 (1981), 1-31.

3. H. Bass, On the ubiquity of Gorenstein rings, Math. Z. 82 (1963), 8-28.

4. D. Buchsbaum and D. Eisenbud, Algebra structures for finite free resolutions, and some structure theorems for ideals of codimension 3, Amer. J. Math. 99 (1977), 447-485.

5. R. Buchweitz, Contributions à la théorie des singularités, Thesis, University of Paris, 1981.

6. __ On deformations of monomial curves, Lecture Notes in Math., vol. 777, Springer-Verlag, Berlin and New York, pp. 205-220.

7. L. Burch, On ideals of finite homological dimension in local rings, Proc. Cambridge Philos. Soc. 64 (1968), 941-966.

8. H. Cartan and S. Eilenberg, Homological algebra, Princeton Univ. Press, Princeton, N.J., 1956.

9. P. De Carli and S. Gabelli, Una classe di algebre rigide, Ann. Univ. Ferrara Sez. VII (N.S.) 25 (1979), 69-74.

10. E. S. Golod, A note on perfect ideals, Algebra (A. I. Kostrikin, ed.), Moscow State Univ. Publishing House, 1980, pp. 37-39.

11. R. Hartshorne, A property of A-sequences, Bull. Soc. Math. France 94 (1966), 61-66.

12. J. Herzog, Certain complexes associated to a sequence and a matrix, Manuscripta Math. 12 (1974), 217-248.

13. __ Deformationen von Cohen-Macaulay Algebren, J. Reine Angew. Math. 318 (1980), 83-105.

14. J. Herzog, A. Simis and W. V. Vasconcelos, Approximation complexes of blowing-up rings, J. Algebra 74 (1982), 466-493.

15. M. Hochster, Properties of noetherian rings stable under general grade reduction, Arch. Math. 24 (1973), 393-396.

16. Topics in the homological theory of modules over commutative rings, CBMS Regional Conf. Ser. in Math., no. 24, Amer. Math. Soc., Providence, R.I. 1975.

17. M. Hochster and J. Eagon, Cohen-Macaulay rings, invariant theory, and the generic perfection of determinantal loci, Amer. J. Math. 93 (1971), 1020-1058.

18. C. Huneke, Linkage and the Koszul homologv of ideals, Amer. J. Math. 104 (1982), 1043-1062.

19. E. Kunz, Almost complete intersections are not Gorenstein rings, J. Algebra 28 (1974), 111-115.

20. A. Kustin and M. Miller, A general resolution for grade four Gorenstein ideals, Manuscripta Math. 35 (1981), 221-269.

21. Algebra structures on minimal resolutions of Gorenstein rings, Commutative Algebra: Analytic Methods (R. Draper, ed.), Marcel Dekker, New York, 1982.

22. Algebra structures on minimal resolutions of Gorenstein rings of embedding codimension four, Math. Z. 173 (1980), 171-184.

23. ___ Constructing big Gorenstein ideals from small ones, J. Algebra 85 (1983), 303-322.

24. Structure theory for a class of grade four Gorenstein ideals. Trans. Amer. Math. Soc. 270 (1982), 287-307.

25. J. Lipman, Free derivation modules on algebraic varieties, Amer. J. Math. 87 (1965), 874-898

26. H. Matsumura, Commutative algebra, Benjamin, New York, 1970.

27. C. Peskine and L. Szpiro, Liaison des variétés algébriques. I, Invent. Math. 26 (1974), 271-302. 
28. M. Schaps, Deformations of Cohen-Macaulay schemes of codimension 2 and non-singular deformations of space curves, Amer. J. Math. 99 (1977), 669-685.

29. B. Ulrich, Gorenstein rings as specializations of unique factorization domains, J. Algebra 86 (1984), $129-140$.

30. J. Watanabe, A note on Gorenstein rings of embedding codimension three, Nagoya Math. J. 50 (1973), 227-232.

Department of Mathematics, University of South Carolina, Columbia, South Carolina 29208

Department of Mathematics, University of Tennessee, Knoxville, Tennessee 37996 